\title{
Emerging barcode particles for multiplex bioassays
}

\author{
Yueshuang $\mathrm{Xu}^{1}$, Huan Wang ${ }^{2}$, Baoan $\mathrm{Chen}^{1 *}$, Hong $\mathrm{Liu}^{2^{*}}$ and Yuanjin Zhao ${ }^{1,2^{*}}$
}

\begin{abstract}
With the increasing demand for multiplex and high-throughput analysis of large numbers of biomolecules, multiplex technology becomes a promising tool for carrying out thousands of individual reactions at the same time for large-scale biological analysis. Among current technologies, suspension arrays based on appropriate barcode particles have been popularly used in multiplex bioassays of many research fields with the ability of unique encoding, such as in the clinical, medicinal, nutritional, and environmental fields. Besides the unique form of barcode, these particles have higher flexibility, better sensitivity, and faster reaction kinetics. In this review, we present some examples of typical barcode particles that are divided into different groups depending on how they are encoded and their applications in multiplex bioassays for different targets such as proteins, DNA and RNA sequences, and cells. The bioassays for monitoring food safety, drug research, and clinical diagnosis are also described.
\end{abstract}

Keywords: barcode, suspension array, bioassay, multiplex, particle

\section{INTRODUCTION}

Given the huge increase in the amount of biomolecular information for research or clinical purposes, there is an increasing need for bioassays to determine the biological activity or specific functional response of a substance. Because of the complexity of biomolecules, the currently available physicochemical approaches are usually insufficient to characterize and analyze their potency $[1,2]$. During this unprecedented growth phase of bioassays, many technologies that encompass applications in the functional analysis of unknown genes, drug discovery, and clinical diagnosis have been developed [3]. Because the multiplexed high-throughput analyses of a large quantity of biomolecules are badly needed now, multiplex technology, which allows multiple discrete assays to be performed simultaneously within the same microvolume sample, is a promising tool for carrying out thousands of individual reactions at the same time for large-scale biological analysis [4-6].

Clinically, the approach to performing multiplex bioassays by dividing single samples into appropriate individual aliquots for simultaneous single target analysis consumes large volumes of material and has difficulties in analyzing large numbers of targets. Thus, one of the most important challenges in developing multiplex bioassays is to track each reaction in a mixture. Perhaps the most popular approach is to use the so-called planar array technology, in which each of the probe molecules is encoded by its exact two-dimensional location on a microtiter plate or on an array [7,8]. Although planar arrays can realize multiplex high-density analysis, there are some disadvantages in the aspect of the strategy by which they are fabricated and used. There is a relatively limited quantity of arrays that can be fabricated simultaneously, and specific probe molecules have to be adhered to each spot of the planar array separately under the same conditions, which may not be suitable for all of them. These drawbacks, as well as the lack of flexibility, sensitivity, and hybridization rate, have limited their applications $[9,10]$.

Suspension array technology using barcode particles as microcarriers has emerged as an alternative. Instead of positional encoding, each particle carries a unique form of barcode that allows identification of the probe molecules attached to it, and the number of codes required depends on the assays, ranging from a few tens to very large numbers [11]. While fluorescently encoded barcode particles have achieved great commercial success $[12,13]$, many other coding technologies have also been proposed for the development of barcode particles, such as graphic, electronic, and chemical encoding methods [14-18]. Unlike planar arrays, probe molecules can be bound to millions of barcode particles at the same time under conditions that are optimal for each probe. In addition, such particles have higher flexibility, better sensitivity, and faster reaction kinetics by efficient mixing in sus-

\footnotetext{
${ }^{1}$ Department of Hematology and Oncology, Zhongda Hospital, School of Medicine, Southeast University, Nanjing 210009, China

${ }^{2}$ State Key Laboratory of Bioelectronics, School of Biological Science and Medical Engineering, Southeast University, Nanjing 210096, China

* Corresponding authors (emails: cba8888@hotmail.com (Chen B); liuh@seu.edu.cn (Liu H); yjzhao@seu.edu.cn (Zhao Y))
} 
pension and can be analyzed in high-throughput microfluidic systems. With these advantages, barcode particles have been applied in a variety of multiplex bioassays in different fields including monitoring food safety, drug discovery, clinical diagnosis, and biodefense [19-24].

Although a number of reviews on barcode particlebased multiplex bioassays have been published because of their good application prospects in many fields, few literatures systematically review their biomedical applications. Moreover, with the increasing investment in research, the development and application of barcode particles in multiplex detection are changing with each passing day. Thus, in this paper, we provide an overall review of encoding technologies that are widely used currently and introduce their latest research and applications in multiplex detection, especially their biomedical applications. Firstly, we mainly introduce the strategies for manufacturing barcode particles, which are divided into several groups based on how they are encoded. Then we focus on studies dealing with the barcode particlebased multiplex bioassays for targets, such as proteins, DNA and RNA sequences, and cells, and their latest applications mainly in biomedical fields, such as clinical diagnosis, food safety and drug research. Finally, we present an outlook for the commercial and technological development of particle-based bioassays.

\section{ENCODING PARTICLES}

Barcode particles are readable tags used widely to track and identify vast amounts of information and are useful in multiplex assays. Classification of these barcode particles relies on different encoding technologies including optical, graphical, magnetic, and shaping methods. Taking the advantages of different encoding technologies, hybrid encoding methods have also been developed for increasing numbers of codes and for a wider range of applications.

\section{Optical encoding}

\section{Organic dye encoding}

Out of the optical encoding barcodes, fluorescent encoding with unique emission spectra and fast readout is the most common encoding method. Moreover, among the fluorescent materials, organic dyes have been the most widely used to fabricate fluorescent barcodes because of several advantages, such as low price and wide range of commercial availability. Although they can be used for various applications, there are also some obvious disadvantages as the encoding element, such as photo- bleaching, limited encoding ability and multi-excitation light used for different dyes [25]. In addition to these disadvantages mentioned before, the aggregation of these organic dyes with high concentration is also a problem during the preparation of barcodes. When it happens, the fluorescent molecues arrange in the form of $\pi-\pi$ stacking which causes aggregation-related quenching and subsequent decrease of spectral intensity. Thus, all these problems restrict their applications as the encoding element.

To overcome the photobleaching problem, the barcodes with inorganic fluorescent dyes inside the silica nanoparticles have been developed. Compared with organic dye encoding barcodes, such barcodes have better photostability [26]. To increase the number of codes, different organic dyes could be mixed by various ratios, for example, one kind of DNA barcode could have 7 different codes by using fluorophores of two colors [27]. As for multi-excitation light uses, single excitation can make the readout easier and inexpensive, thus for this purpose, fluorescence resonance energy transfer (FRET) has been developed to prepare barcodes with multiple emissions under one single excitation [28]. In this process, because of their spectral overlap, three kinds of organic dyes (fluorescein isothiocyanate (FITC), rhodamine 6G and carboxy-X-rhodamine) were employed to realize FRET. Fig. 1a shows the organic dye encoding barcodes with FRET-mediated codes, and Fig. $1 \mathrm{~b}$ shows the excitation and emission peaks of the three organic dyes. In addition, by varying the ratio of these dyes, the barcodes with different emission signals have multi-colors under a single excitation. With the advantages of high photostability, well-controlled sizes and good biocompatibility, such barcodes could be used to trace proteins, nucleic acids and other molecules within living systems.

In order to overcome the problem of aggregation-related quenching, a new kind of active dyes was developed to enhance the fluorescence intensity [29]. The reason why such dyes could enhance the fluorescence intensity when aggregation happened was that the special structure of these dyes could restrict the occurrence of $\pi-\pi$ stacking. Currently, this kind of active dyes, such as 1,8-naphthalimide derivative fluorophore and 10-cetyl- $10 \mathrm{H}$-phenothiazine-3,7-(4,40-aminophenyl)-acetonitrile, were used to prepare different barcodes. Because they could be uptaken by HeLa cells, they also have potential for biological applications.

\section{Quantum dot encoding}

Quantum dots (QDs) are usually semiconductor nanocrystals composed of elements of groups on the periodic 

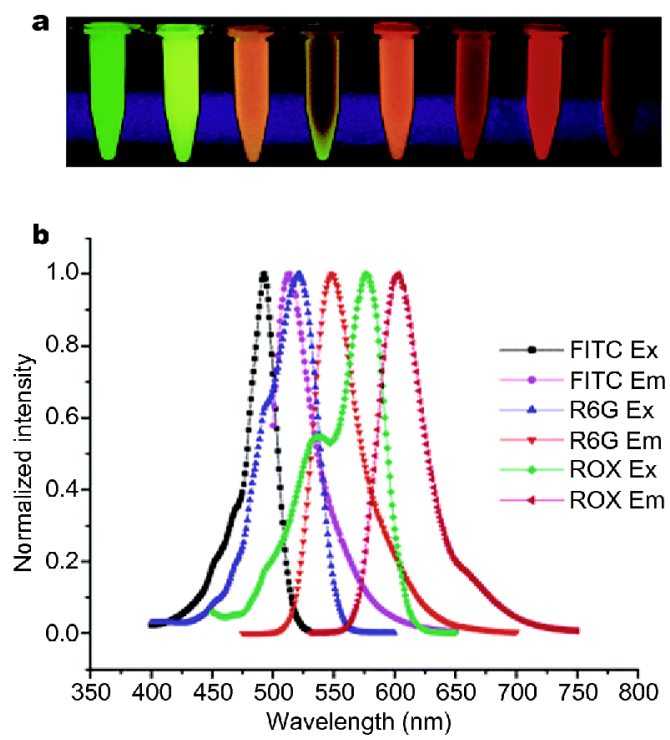

c
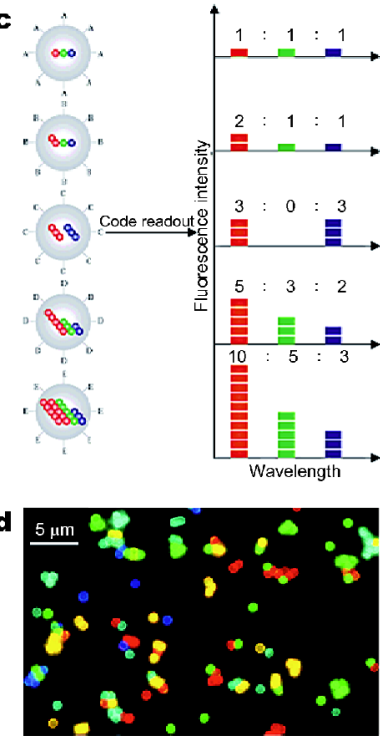
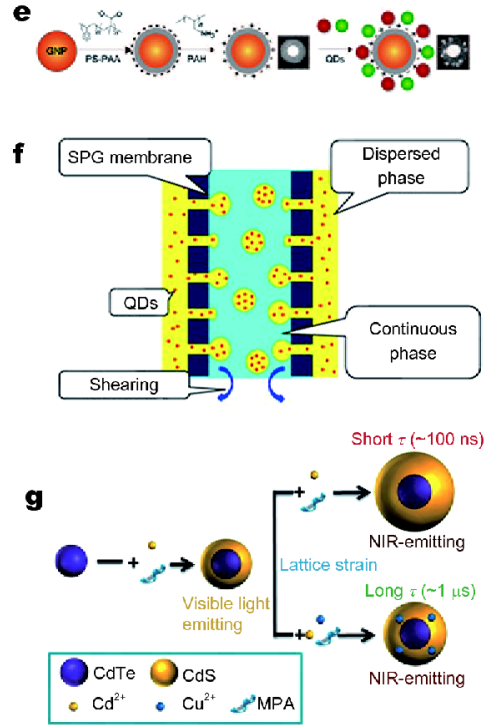

Figure 1 (a) Organic dye encoding barcodes with FRET-mediated codes. (b) The excitation and emission peaks of the three organic dyes. (c) Schematics of different QDs encoding with multiplex wavelength and intensity. (d) Fluorescence micrograph of different QD-encoded barcode particles on the polylysine-coated glass slide with the emission wavelengths of 484, 508, 547, 575, and $611 \mathrm{~nm}$, respectively. (e) Fabrication process of gold nanoparticles adsorbed with QDs by using layer-by-layer method. (f) Schematics of developing size-controllable QD-encoded barcodes with the MESE strategy. (g) Synthesis of QDs with NIR emission as well as long lifetime by integrating lattice strain and doping. (a, b) Reprinted with permission from Ref. [28]. Copyright 2006, American Chemical Society. (c, d) Reprinted with permission from Ref. [30]. Copyright 2001, Nature Publishing Group. (e) Reprinted with permission from Ref. [35]. Copyright 2012, Wiley-VCH. (f) Reprinted with permission from Ref. [40]. Copyright 2013, American Chemical Society. (g) Reprinted with permission from Ref. [45]. Copyright 2014, Wiley-VCH.

table, such as CdSe, InAs, PbSe and CuInS 2 . They have unique and excellent optical properties as an encoding element, including photostability, high brightness, narrow and tunable emission spectra, and single wavelength excitation which can excite different emission spectra of QDs. The emission spectra tuned by the sizes of QDs can offer a dozen of different visible colors (400-800 nm). By using QDs of different emission colors and different intensity levels, multiplex codes (more than one million in theory) can be generated, with the following encoding capacity calculation: $C=N^{m}-1$ ( $C$ is the number of codes; $N$ is the number of emission intensity levels and $m$ is the number of colors) (Fig. 1c) [30]. But as a matter of fact, there exist some problems of QDs encoding in the application of multiplexed assays. For example, the overlap between different intensities usually limited their encoding capacity, and the reporter is still needed when the spectral region is not attainable for encoding.

Many approaches for preparing QD-encoded barcode particles have been developed by three technologies: (i) embedding of QDs into the polymer particles directly, in which the pre-synthesized particles and QDs are scattered in the swelling/infiltrating solution firstly, and then the QDs infiltrate into the particles and finally stay in the subsurface of the particles [31,32]. This method was first introduced by Nie et al. (Fig. 1d) and is by far the most widely used. (ii) Gathering of QDs on the surface of particles by layer-by-layer method [33-36]. In this method, QDs are adsorbed consecutively onto the particles with opposite charge or with the coordination interaction and affinity interaction between the functional particle and QDs, and thus the number and color of QDs cycles would determine the optical properties of barcode particles simply (Fig. 1e). (iii) Combination of QDs with the particles during the particle synthesis, according to encapsulation or chemical grafting $[37,38]$. By the covalent bond between QDs and polymer hosts, the polymer hosts can contact QDs directly and the barcodes have long-term stability.

In spite of great progress, some remarkable challenges remain about the fabrication of QD-encoded barcode particles, such as inferior stability and size control. A selfhealing encapsulation approach has been developed to prepare QD-encoded barcode particles with high-stability and uniformity [39]. In this study, due to the interaction of molecular links and heat motion, QDs could be encapsulated with uniform distribution in the self-healing process of porous polymer particles. It was also demon- 
strated that these barcodes were really suitable for quantitative multiplexed analysis because of high stabilities and large QDs loading capacity. Another limitation of the technologies to prepare the QD-encoded barcodes is the size control. This problem could be solved by a membrane emulsification-solvent evaporation (MESE) strategy (Fig. 1f) [40]. In this strategy, the droplets were of good size control because of shirasu porous glass (SPG) membrane emulsification which can uniformize the emulsions effectively. Thus, by changing the sizes of membrane pores, the size-controllable QD-encoded barcodes with different sizes could be fabricated easily. Sizecontrollable and uniform barcodes could also be produced through microfluidic technology $[41,42]$.

Moreover, most reported QD-encoded barcodes have the emission of visible light instead of near-infrared (NIR) light (650-900 $\mathrm{nm})$, whereas the spectrum region of NIR is important for in vivo biological imaging and can avoid fluorescence quenching problem [43], resulting in fairly strong background interference from Rayleigh scattering and autofluorescence of biomolecules and the problems of fluorescent overflow and overlapped composite signals [44]. Finally, the lifetime of QDs could be considered as another important encoding parameter and is generally in the range of one to a few tens of nanoseconds. Thus, it is highly desirable to develop QDs with NIR emission as well as long lifetime. The approach for developing such QDs has been realized by integrating lattice strain and doping, resulting in desired NIR-emitting and long-lifetime of $1 \mu \mathrm{s}$ (Fig. 1g) [45]. Moreover, the NIR-emitting codes with different emission and lifetime could further open new perspectives for the development of optical encoding.

\section{Upconversion nanoparticle encoding}

As mentioned above, the background interference and other problems caused by light in short-wavelength region would severely limit the detection of reporter signals. In order to avoid such interference to guarantee the detection accuracy, it is necessary to separate the code signals from background signals and reporter signals. There is almost no NIR light-induced background fluorescence or reporter signals because of little interaction between them. Meanwhile, the code signals of upconversion nanoparticles (UCNPs) only can be excited by NIR light instead of UV or visible light into visible emission [46,47].

This characteristic is given by lanthanides ions, and they are usually doped into the host materials that serve as activators or sensitizers [48]. During the NIR excitation, the sensitizers $\left(\mathrm{Nd}^{3+}\right.$ or $\left.\mathrm{Yb}^{3+}\right)$ absorb energy and then transfer it continuously to the activators $\left(\mathrm{Ho}^{3+}, \mathrm{Er}^{3+}\right.$ or $\left.\mathrm{Tm}^{3+}\right)$. After receiving energy from the sensitizer, the activator could emit one UV or visible photon while more NIR photons have been absorbed by the sensitizer $[49,50]$. Due to this unique ability, UCNPs exhibit exceptional great properties with long luminescence lifetime, sharp emission bands and low toxicity, and the polychromatic emission also can be tuned by changing the lanthanides ions. Thus, UCNPs are suitable for multicolor encoding and the benefits of UCNPs have been exploited (Fig. 2a) [51-55].

The fluorescence spectra of UCNPs-encoded barcodes can be obtained after NIR excitation as shown in Fig. $2 \mathrm{~b}$. By varying the doping amount and UCNPs components, barcodes with unique spectrum can be developed [56]. The number of codes can increase exponentially when both components and intensities are changed simultaneously. In general, $N$ intensiy levels with $m$ colors can generate $\left(N^{m}-1\right)$ different codes, which is similar to that of QDs. Besides the encoding capacity, a good code requires less spectral interference with fluorescent dyes normally used for bioassays (e.g., Cy3, FITC, Cy5). By using multi-color UCNPs-encoded barcodes that were modified with oligo probes, the use of UCNPs-encoded barcodes for multiplexed bioassays was verified (Fig. 2c) [57]. In this system, different target DNAs are labeled with unique fluorescent dyes and can be identified with the unique spectrum of barcodes. It is worthy to mention that the NIR light indeed only excites the UCNPs, whereas the visible light only excites the fluorescent dyes, resulting in no optical cross talk between them and a wide selection of labels which could serve as another encoding element [58].

Typically, because of organic solvents during the synthetic process, these UCNPs are filled with hydrophobic ligands which limits their biological applications. Therefore, surface hydrophilization is a condition precedent for conjugation between UCNPs and biomolecules [59]. Currently, there have been some good surface modification methods, such as ligand oxidation or exchange reaction, intercalation with amphiphilic polymers, coating with cross-linked polymer and so on [60-65]. However, these methods usually cannot form the stable protective shell around the UCNPs to protect them from the destruction of solvent molecules and fluorescence quenching [66]. Embedding UCNPs into polymer particles represents a promising method to form hydrophilic surface as well as a solid protective shell which is impassable to solvent molecules [67]. Therefore, the efforts to incorporate UCNPs with polymer particles have been in- 

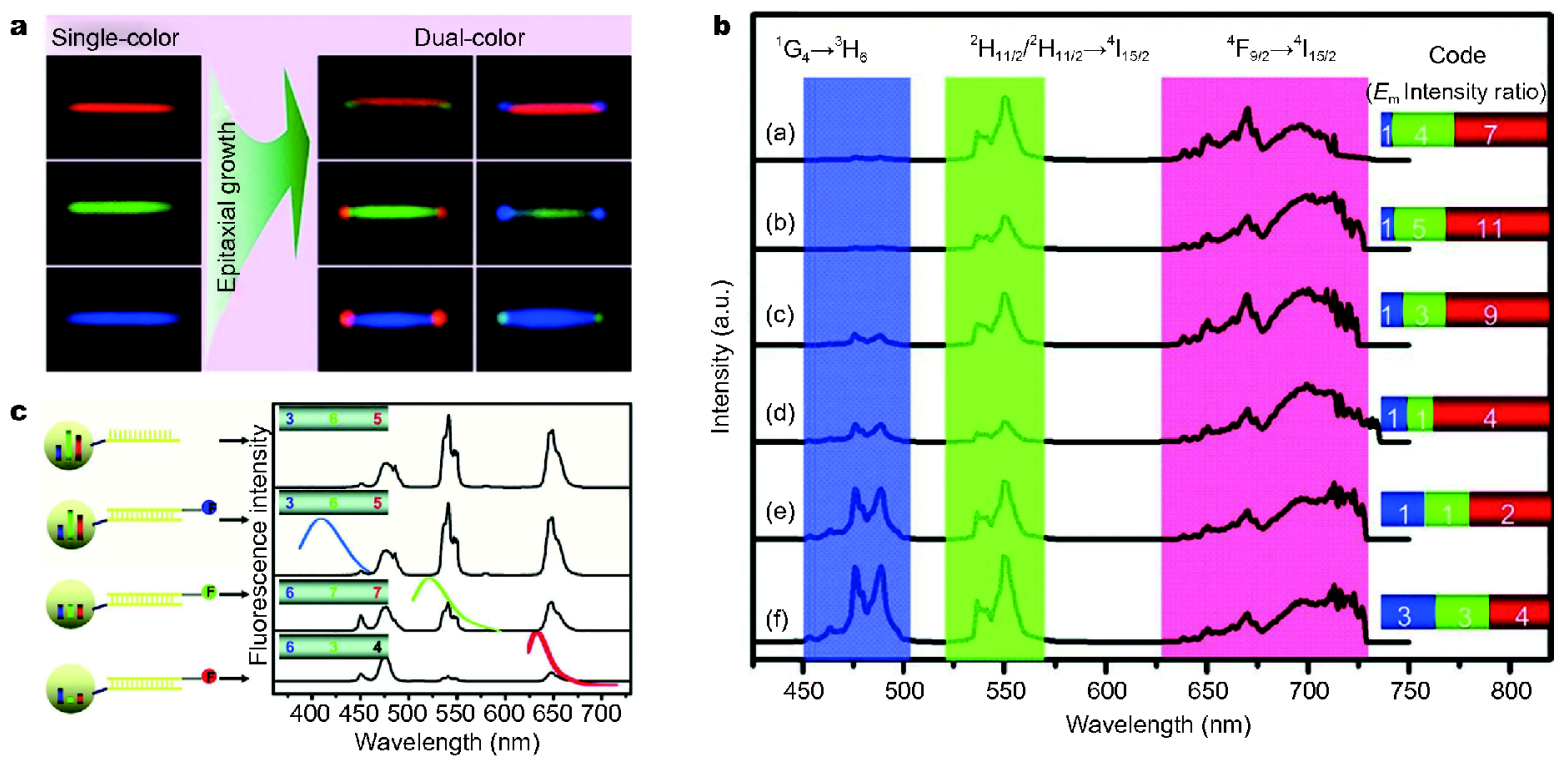

Figure 2 (a) UCNPs-encoded nanorods with multi-color by combining three different primary colors. (b) The most commonly used encoding principle of UCNPs-encoded barcodes by utilizing different colors and various intensity levels. (c) Schematic illustration and test results of multiplex bioassay by employing oligo probe-modified UCNPs-encoded barcodes. (a) Reprinted with permission from Ref. [52]. Copyright 2014, American Chemical Society. (b, c) Reprinted with permission from Ref. [56,57]. Copyright 2011, Wiley-VCH.

vestigated by several groups to realize bio-applications.

\section{Structural color encoding}

Structural color is produced by the modulation or interference of light due to a structured surface [68-71]. One of the structural colors can be generated through photonic crystals (PhCs) that are common in pearls, peacock feathers and so on [72-74]. The structural colors of PhCs derive from their periodically arranged structures that can control and manipulate the diffraction of light. Although many optics-encoded barcodes have been developed for multiplexed assays in recent years, PhCs with unique advantages are more attractive today [75-84].

$\mathrm{PhCs}$ have a periodically modulated dielectric constant and a photonic bandgap [85-87]. Light in the bandgap is usually reflected instead of propagating directly through the materials, and thus create the different structure color codes as can be seen in Fig. 3a [75]. Their dynamic diffraction state can be described by Bragg equation:

$$
m \lambda=2 n d \sin \theta,
$$

where $\lambda$ is the reflection peak of light, $m$ is the diffraction order, $n$ is the average refractive index, $d$ is the diffraction planar spacing and $\theta$ is the Bragg glancing angle. According to the Bragg equation of photonics mentioned before, the reflection peak of the light could be changed along with the distance between two neighboring nano- particles or the refractive index of PhC barcodes. Thus, $\mathrm{PhC}$ barcodes with the unique structures and coding capacity of different colors can be used in wide areas of optics, such as wetting, sensing, catalysis, electrodes, anticounterfeiting and so on [88-100].

Some novel encoding technologies have been developed in recent years. To achieve higher number of codes, the primary structural color from the PhCs has been combined in different ratios to result in secondary colors as shown in Fig. 3b [101]. Bilayer films can be prepared by infiltrating silica-ETPTA solution on a layer pre-fabricated before and finally polymerizing it. Such $\mathrm{PhC}$ bilayer films could have unusual colors because of two different layers of colors, for example, blue with green would turn cyan, green with red would turn yellow, and red with blue would turn magenta, respectively. All the PhC multilayer films, including the transparent trilayer films, can also be fabricated by using this method, and they have more than one distinct reflection peaks from each layer of the films without deterioration, respectively. The multicolored $\mathrm{PhC}$ patterns with multiple reflection peaks have been demonstrated to be ideal coding carriers in a number of applications, such as multiplexed assays and anti-counterfeiting. In addition, because of the tunable unique peaks of nanoporous anodic alumina (NAA) between 300 and $700 \mathrm{~nm}$, it also has been used as PhC-encoded barcodes [102]. In the 8-bit system with the spectral signal of 

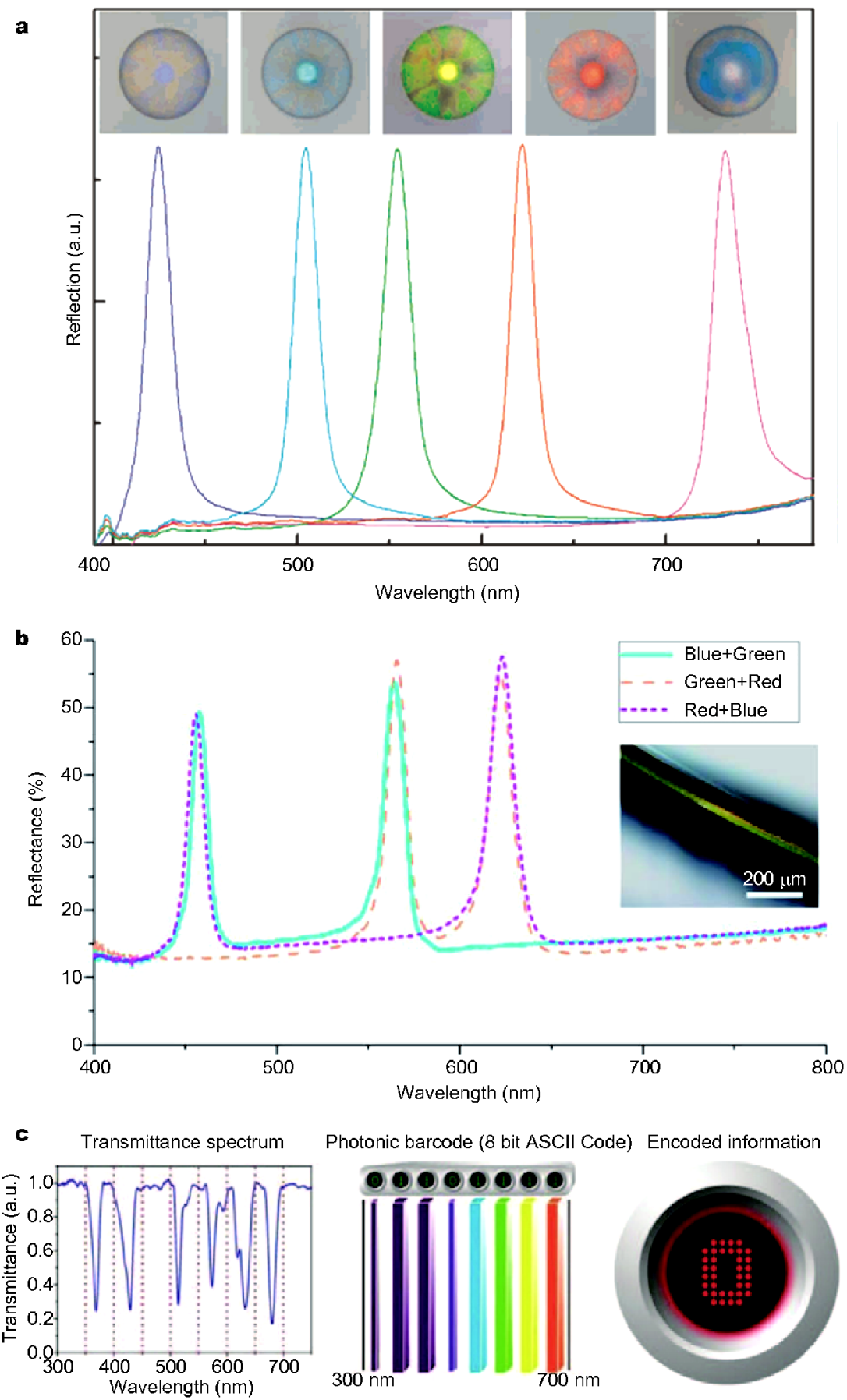

Figure 3 (a) Optical microscopy images and reflection spectra of five kinds of PhC barcode particles. (b) Reflectance spectra of three bilayer films and each shows two distinguishing peaks. The inset is the cross-section of the bilayer film. (c) Optical encoding of information within the transmittance spectrum of NAA PhCs and their conversion into photonic barcodes (example $01101111=$ 'o' in 8-bit ASCII system). (a) Reprinted with permission from Ref. [75]. Copyright 2008, American Chemical Society. (b) Reprinted with permission from Ref. [101]. Copyright 2013, American Chemical Society. (c) Reprinted with permission from Ref. [102]. Copyright 2016, the Royal Society of Chemistry.

NAA as encoding information, there are total 256 distinct colors can be created with the consideration of two states: ON (1) and OFF (0) per bit (Fig. 3c).
$\mathrm{PhC}$ barcodes have been widely used as coding carriers in multiplex bioassays [103-105]. As surface enhanced Raman spectroscopy (SERS) has become a promising 
technology in the application of multiplexed assays, PhCs with periodically arranged structures and good fabrication reproducibility can also be good SERS substrates [106]. In addition to bioassays, PhCs are also used for recognition of various ions and solvents either by using ssDNA probes as sensing units or by applying the swelling property of hydrogel $\mathrm{PhC}$ barcodes, resulting in the shifts of the spectra [107-110].

\section{SERS encoding}

Raman spectroscopy is a spectroscopic technology used for analyzing molecules by observing vibrating, rotating, or other low frequency modes. It depends on Raman scattering of homogeneous light whose wavelength is in the range from near ultraviolet to NIR. Raman scattering occurs due to the up or down shift of monochromatic light based on the inelastic scattering due to the vibration or rotation of molecules. It is difficult to detect the scattering because it has very low efficiency. In order to improve the Raman scattering efficiency, SERS has been studied by employing noble metal detection surfaces, including silver, copper or gold layer and a widely used optical encoding element in biological application [111-113].

As an encoding element, SERS has some characteristic advantages, such as: (i) greater sensitivity with the detection capability of single-molecular level; (ii) no photobleaching and high photostability; (iii) narrow spectra bandwidth; (iv) high capacity of multiplexing with the fingerprint characteristics. In addition, the unique spectra of different Raman codes only need a single laser excitation. In recent years, SERS signals have been employed as not only encoding carriers but also labels because of their great encoding capability and ideal sensitivity in suspension array technology. Normally, SERSencoded barcode particles comprise a plasmonic nucleus responsible for the generation of the electric field, a Raman reporter for the unique vibrational fingerprint and a coating layer which can prevent the code from leaching out, and provide a convenient surface for further chemical functionalization and so on. There have been several synthetic strategies reported to fabricate SERSencoded barcode particles, including the adsorption of Raman reporters and the particle coating [114,115].

However, because of the increasing amount of desired codes, Raman reporters that are employed for the encoding may become rather complicated, whereas it is very difficult to distinguish them well because of their similar chemical structures. The above problems lead to limited number of codes that can be obtained and therefore the enhanced encoding capability is still desired. A wave- number-intensity related SERS-signatures encoding approach was developed, in which silver nanoparticles (Ag NPs) were employed as the substrate, meanwhile 4methoxythiophenol (4-MT), 2-naphthalenethiol (2-NAT) and 1,4-benzenedithiol (BDT) were chosen as Raman reporters (Fig. 4a) [116]. By using different reporters and changing their molecular ratio, numerous different unique SERS signatures of different wavenumber and intensity ratio could be obtained. This method demonstrated that the encoding capability of SERS is dependent on the kind of Raman reporters as well as their molecular ratio, as another influence factor. Although the encoding capacity could be increased by incorporating different SERS reporters with different ratios, it is very important to control the amount of involved reporters precisely in the fabrication process. Moreover, due to more and more reporters used in the fabrication process, it may be quite hard and time-consuming to fabricate the ideal SERS-encoded barcodes. Thus, the dual mode encoded barcodes have been studied by employing both various fluorescent and Raman reporters to obtain a greater encoding number (Fig. 4b) [117,118]. Among them is the organic-metal-QD hybrid barcodes, whose encoding elements are joint SERS-fluorescence signals with more distinguished spectral codes. Moreover, in comparison with the SERS encoding approach mentioned above, the fabrication process of this method is easier and less time-consuming even when the number of reporters is increased, because these two kinds of reporters are assembled onto two separate layers.

Moreover, Raman spectroscopy also can be used to characterize the pathology-related biochemical properties of biological tissues. Usually, because of the same spectral region where both fluorescent and Raman signatures come out simultaneously, the Raman spectral signal could be badly interfered by the fluorescent spectrum, resulting in a hard readout of Raman signal. To overcome this, signal processing methods have been reported to resolve the Raman signal from fluorescence interference, which shows that it is feasible to read absolute Raman signals of biological tissues without the strong fluorescent interference [119].

\section{Graphical encoding}

Graphical encoding relies on the pattern of optical elements on a microcarrier, including striped rods, ridged particles and dot-patterned particles. Due to the amazing photo-electric properties of metal materials, one kind of graphical encoding method is using striated metal nanorods, composed of different metals such as gold (Au), 

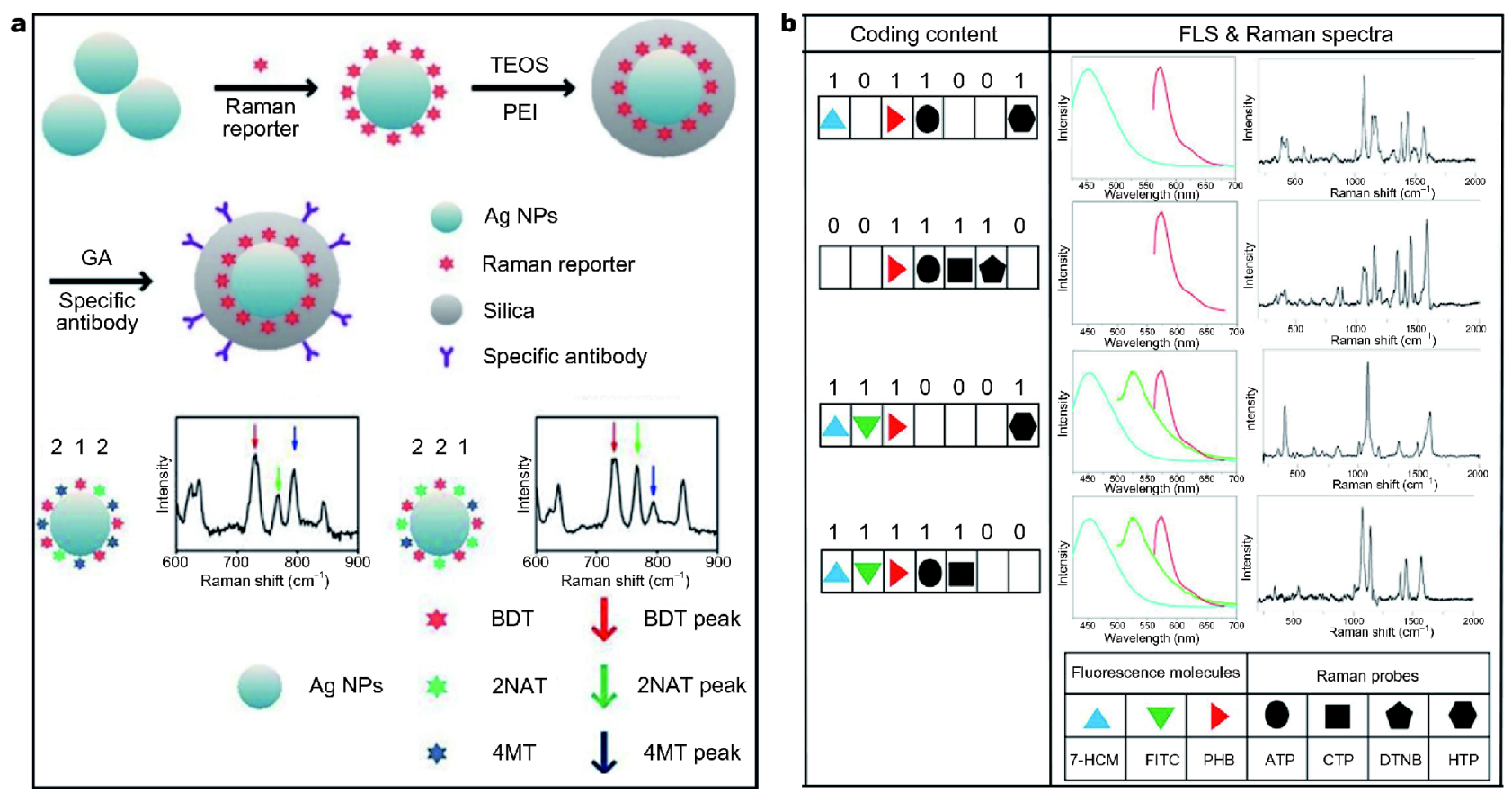

Figure 4 (a) Schematic of wavenumber-intensity related SERS-signatures encoding approach, and the different unique SERS signatures of different wavenumber and intensity ratio. (b) Fluorescence spectra and Raman spectra of the dual mode encoded barcodes. (a) Reprinted with permission from Ref. [116]. Copyright 2014, the Royal Society of Chemistry. (b) Reprinted with permission from Ref. [117]. Copyright 2016, American Chemical Society.

cobalt $(\mathrm{Co})$, copper $(\mathrm{Cu})$, and so on [120]. The metal stripe pattern formed with the help of an electrodeposition method in the preparation process is used to encode information and such information could be identified easily by using reflectance microscopy dependent on the different reflectivities of metal materials (Fig. 5a). For example, Au has high reflectivity in the blue, making the $\mathrm{Au}$ segment appear more reflective than the silver segment of the nanowires. Furthermore, the composition of the alloy nanowires can be characterized using X-ray fluorescence (XRF) as well as electrochemical readouts $[121,122]$. The graphical encoding technique can generate high number of barcodes, for example, the $5-\mathrm{mm}$ rods composed of two kinds of metals can possess up to 10 stripes if the minimum stripe length could be about $500 \mathrm{~nm}$, which corresponds to 528 codes. In addition, the code number could be increased by increasing the rod length or the metal types as well as varying the length of the segment. However, it may be unrealizable to fabricate a lot of characteristic nanorods with electrodeposition approach, because only one type of nanorod could be fabricated each circle.

Another kind of graphical encoding technology is using a pattern of holes which was firstly reported by 3D Molecular Sciences Ltd. [123]. The surrounding hole patterns are employed to show the direction of barcodes, and the residual holes would form the encoding information of barcodes. Such barcodes have great encoding capability with $2^{35}$ codes. A similar encoding technology has been demonstrated and the difference is that the demonstration of encoding, reaction process and readout of the signals are all completed in a microfluidic system [124]. As shown in Fig. 5b, two flows of poly(ethylene glycol) (PEG) monomer run together separately through a microfluidic system with laminal channels. One of the two flows is the fluorescence-labelled monomer that is patterned by UV light as the encoding information, while the other flow is full of monomers as well as probes used in the bioassays. The region surrounding the encoded holes is fluorescent that can increase the signal-noise ratio and identify the encoding information simultaneously when the analysis has been completed.

Lithographic methods also allow the fabrication of graphically encoded particles with novel encoding patterns [125]. A representative example of colorful barcode particles has been developed by combining the readable spectral encoding with the distinguishable graphical encoding (Fig. 5c) [126]. In this study, eight very different colors are used to realize multilevel encoding, resulting in geometric growth of the encoding capability with the 

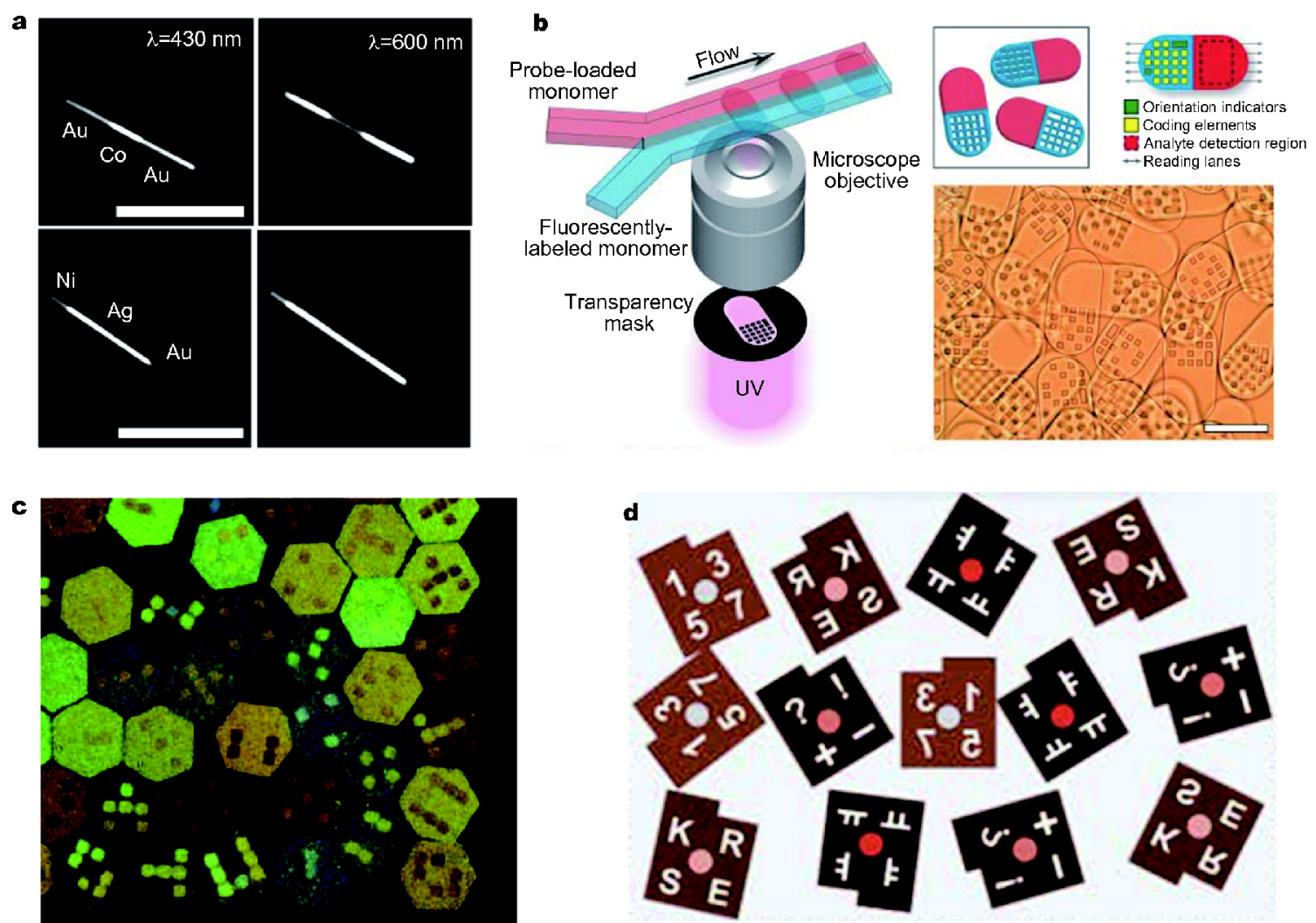

Figure 5 (a) Reflectivity of nanorods composed of $\mathrm{Co}, \mathrm{Au}, \mathrm{Ni}$, and Ag. (b) Schematic of manufacturing particles in microfluidic system. One of the two flows is the fluorescence-labelled monomer that is patterned by UV light as the encoding information, and the other flow is full of monomers as well as probes used in the bioassays. (c) Colorful barcode particles with readable spectral encoding and the distinguishable graphical encoding. (d) Graphically encoded barcode particles with four characters. (a) Reprinted with permission from Ref. [120]. Copyright 2003, American Chemical Society. (b) Reprinted with permission from Ref. [124]. Copyright 2007, American Association for the Advancement of Science. (c) Reprinted with permission from Ref. [126]. Copyright 2010, Springer Nature. (d) Reprinted with permission from Ref. [127]. Copyright 2017, the Royal Society of Chemistry.

comparison to conventional binary barcode particles. For example, it usually has only 210 distinguishable codes in 10 different bits of binary barcodes and there are $8^{10}$ easily readable color codes in such colorful barcode particles. Fig. $5 \mathrm{~d}$ also shows a novel platform that combines the high coding capacity with the easy-to-read colorimetry appropriate for an assay, in which $900 \mu \mathrm{m}$ barcodes are encoded with four different kinds of symbols, including numbers, alphabets, punctuations and so on. The barcodes with about two million codes can be covalently linked with adhesive molecules [127]. The use of this platform to realize the colorimetric bioassay of analytes has been demonstrated and it also can be really employed for many applications including autoantibody test.

\section{Shape encoding}

Shape encoding uses different shapes to generate the codes. In comparison with other encoding approaches, the shape-encoded barcodes do not have the problem of fluorescence quenching or spectral interference. Silica nanotubes (SNTs) have been synthesized with different hollow structures and shapes from different synthesis templates to create 4 distinct codes, as shown in Fig. 6a [128]. In this study, the shape-encoded SNTs were fabricated through the multistep anodised aluminium template and the length of every fragment was determined by the duration of twice anodic treatment. The SNTs with the readable shapes were easily distinguishable with the only use of traditional optical microscopes. Moreover, SNT with more than four fragments could provide more than 1,000 codes and they are being studied to improve this shape-coded SNT system for multiplexed bioassays.

The second kind of shape-encoded barcodes are usually fabricated by using optofluidic maskless lithography (OFML). This photolithographic methodology naturally enables high-throughput mass-production, which means thousands of identically shaped barcodes can be produced in a single lithographic exposure [129]. By using this 

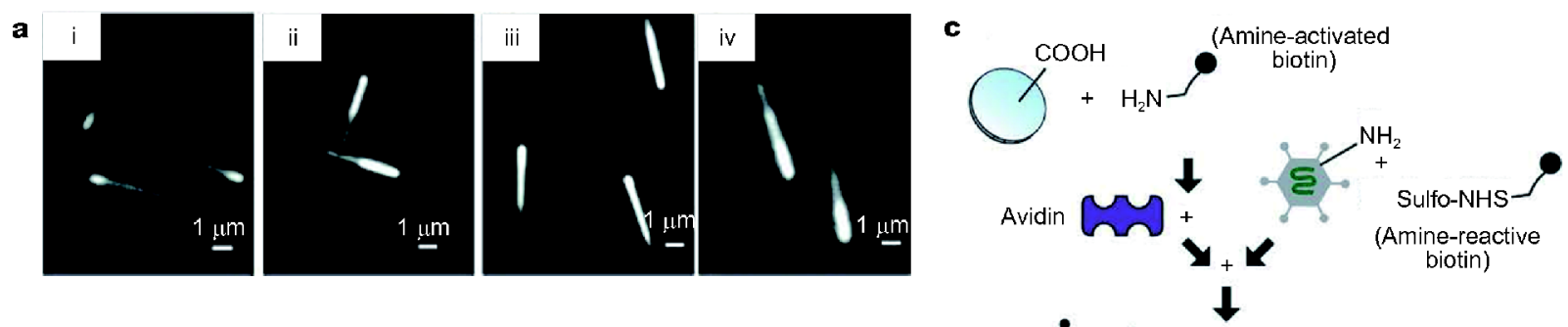

b
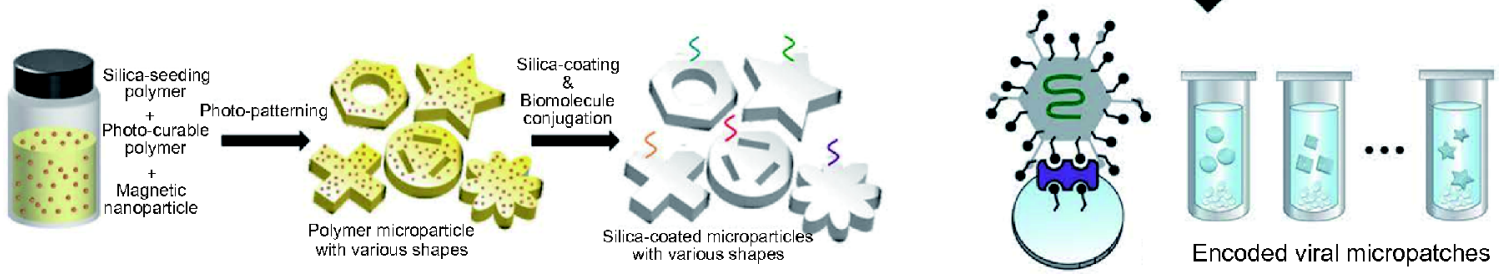

Figure 6 (a) Optical microscopy images of silica nanotubes with different hollow structures and shapes from different synthesis templates. (b) The fabricating process of shape-encoded silica-coated barcodes. (c) The process of immobilizing adenoviral vectors on the shape-encoded viral micropatch and fabricating shape-encoded viral micropatches. (a) Reprinted with permission from Ref. [128]. Copyright 2006, American Chemical Society. (b, c) Reprinted with permission from Ref. [130,131]. Copyright 2015 and 2017, the Royal Society of Chemistry.

method, new shape-encoded silica-coated barcodes have been studied as shown in Fig. 6b [130]. These barcodes with polymer microstructure not only have stable silicon dioxide coating, but also have strong encoding capability for multiplexed bioassays. Another "shape-encoded viral micropatch" also has been developed for convenient localization of gene delivery in multiplex, high-content cellular assays (Fig. 6c) [131]. The conditional transduction of biotinylated adenoviral vectors allows strict confinement of adenoviral gene delivery under the micropatches, and the micropatch is also suitable for image-based assays after the gene delivery, but not so much for performing molecular analysis, by collecting cells after the assays.

However, the shape encoding technique cannot generate numerous distinct and unique barcodes as compared to other encoding techniques. Moreover, decoding for most of the shape encoding involves equipment that requires complex operating procedures and trained personnel to measure the shape of the nano-barcode.

\section{Magnetic encoding}

Magnetic encoding relies on the magnetic properties of nanoparticles (usually $\mathrm{Fe}_{3} \mathrm{O}_{4}, \mathrm{Fe}_{2} \mathrm{O}_{3}$ and $\mathrm{CrO}_{2}$ ). Due to their several advantages, magnetic encoding becomes a natural choice $[132,133]$. Firstly, the magnetic characteristic is particularly stable for a long time without the affection from chemical reagent and exposed light. Secondly, both the stray magnetic field of particles and the external field employed for remotely manipulating particles, are not tested in the watery biotic environment.
Finally, magnetic background in biological samples is minimal.

Multisegmented metallic nanowires have been aroused by conjunction with optical coding techniques as shown in Fig. 7a [134]. Ferromagnetic materials possess a magnetization value that can be measured using vibrational sample magnetometry (VSM). And it is feasible to change their magnetic characteristics by varying the composition of many ferromagnetic materials. Moreover, by replacing a nonmagnetic fragment $(\mathrm{Au})$ of nanowires with one kind of magnetic substances, both optical and magnetic signals from the same nanowire could be obtained simultaneously. Thus, three unique groups that combine different magnetic compositions with $\mathrm{Au}$, including Co-rich/ $\mathrm{Au}, \mathrm{CoFe}$-equiatomic/Au and $\mathrm{Fe}$-rich/Au can be realized, resulting in different shifting position in the hysteresis curves and magnetization value under VSM measurement as encoding elements.

A remote encoding project for micron-sized multi-bit magnetic labels that utilizes magnetic digital planar tags as carriers for biomolecules, and micron-sized magnetic barcodes to encode a unique signature on each label for performing multiplexed high-throughput bioassays has been presented $[135,136]$. The general design of a digital planar tag is shown in Fig. $7 \mathrm{~b}$ : multi-bit magnetic elements with individually different coercivity for remote signature encoding, a gold attaching pad for immobilizing the probes and a backbone made of fully cross-linked SU8 polymer for high chemical resistance. By changing the aspect ratio of the magnetic elements that controls the shape aeolotropism of micrometer sized particles, mag- 

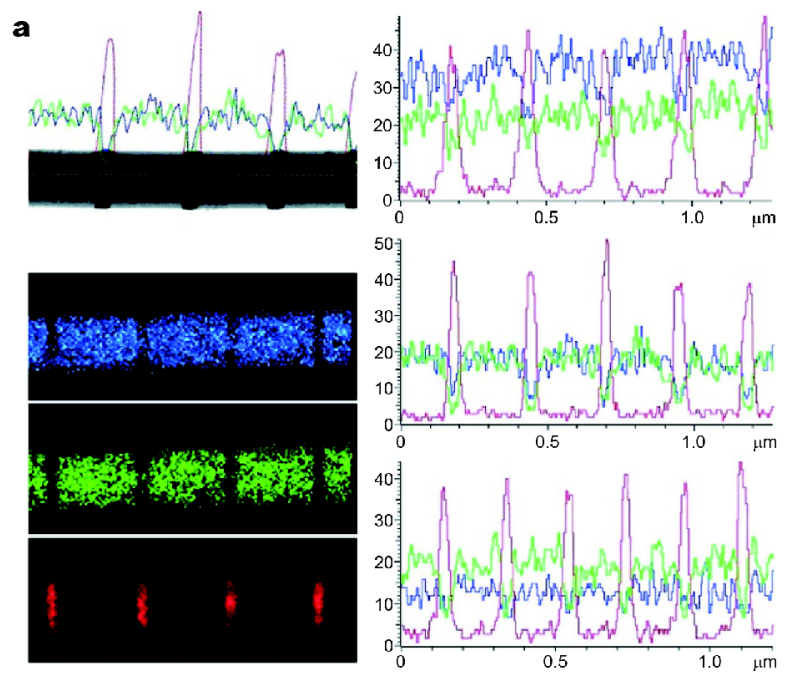

b
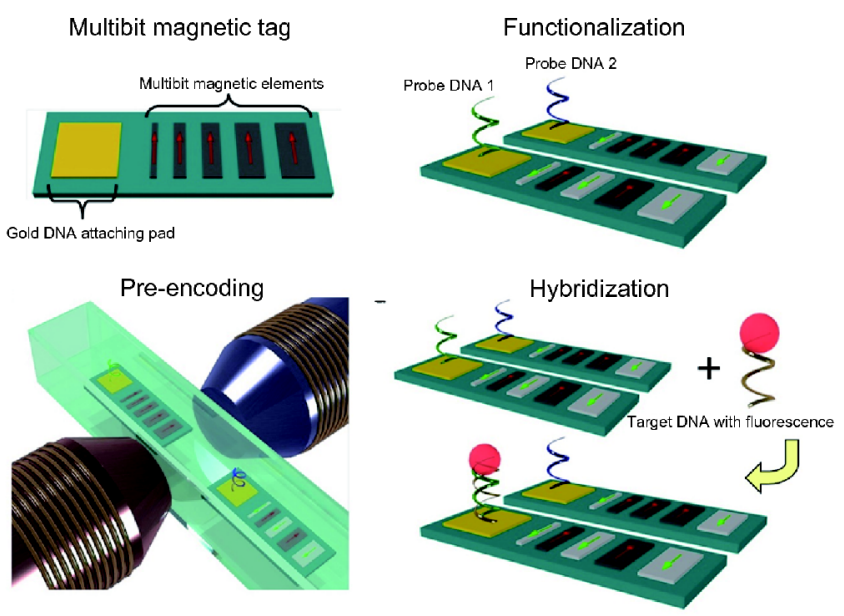

Figure 7 (a) TEM image of a multisegmented metallic nanowire and elemental line scanning results of three different nanowires with different magnetic compositions. (b) General design of a digital planar tag. (a) Reprinted with permission from Ref. [134]. Copyright 2012, Japan Society of Applied Physics. (b) Reprinted with permission from Ref. [135]. Copyright 2008, American Institute of Physics.

netic barcodes exhibit individually different coercivities to an applied external field. The encoding capacity of magnetic barcodes with a vast number of magnetic bits is potentially very huge, and with the use of microfluidic device, the targeted analytes could be analyzed parallelly in a very short time. Another attractive feature of magnetic barcodes is that they will have fluorescence property by incorporating different fluorescent elements into them to further increase the number of magnetic encoding.

\section{Hybrid encoding}

In order to take advantages of each encoding technology and increase the encoding number of barcodes, it is desirable to combine different encoding technologies in a barcode (called hybrid encoding). With more number of codes that are desirable for multiplex bio-applications can be generated, hybrid encoding is the most popular for multiplex bioassays in recent years. Moreover, with different types of covert codes, the combination of the encoding techniques can also be used for multi-layer security in the anticounterfeiting field. The most common hybrid encoding method is to combine fluorescent encoding method, such as organic dyes and QDs with shaped, structural color, Raman or magnetic encoding methods [137], among which the fluorescent-SERS or fluorescent-magnetic dual encoding technology has been described before. As shown in Fig. 8a, the combinatorial multiplex code technology was reported based on the use of QDs and PhC barcodes [138]. In this method, hundreds of codes were obtained by combining fluorescence intensity and spectra of QDs with the reflection spectra of $\mathrm{PhC}$ barcodes. Unlike layer-by-layer (LBL) assemblely of QDs on the surface of PhC barcodes or in-situ polymerization of acrylamide gel containing QDs in the photonic barcodes, this method seems more stable. In addition, based on the advantages of magnetic codes and QDs codes, magnetic-fluorescent hybrid-encoded barcodes have been fabricated by employing poly(styrene/ acrylamide) nanobeads, nano- $\gamma-\mathrm{Fe}_{2} \mathrm{O}_{3}$ and QDs [139]. This kind of nano-sized hybrid-encoded barcodes with both characteristics of fluorescent and magnetic encoding have the advantages of outstanding monodispersity and large encoding capability. Another new hybrid-encoded barcode particle that possesses the strawberry-like structure has also been studied $[140,141]$. In this barcode, large numbers of nanoparticles doped with dyes are tightly attached on the surface of QDs-encoded particles. In comparison with other multi-color barcode particles, this barcode not only has the outstanding capacity of encoding, but also can well avoid optical interference from two kinds of encoding by separating them into different layers. This property makes it convenient for the following accurate decoding (Fig. 8b).

\section{PRACTICAL APPLICATIONS OF BARCODE PARTICLES}

Different barcode particles can be used to detect various analytes for different practical applications. In the following sections, we introduce different detection methods using these barcodes for different analytes and compare 
a
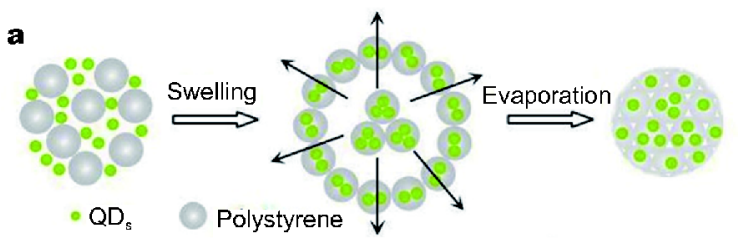

b

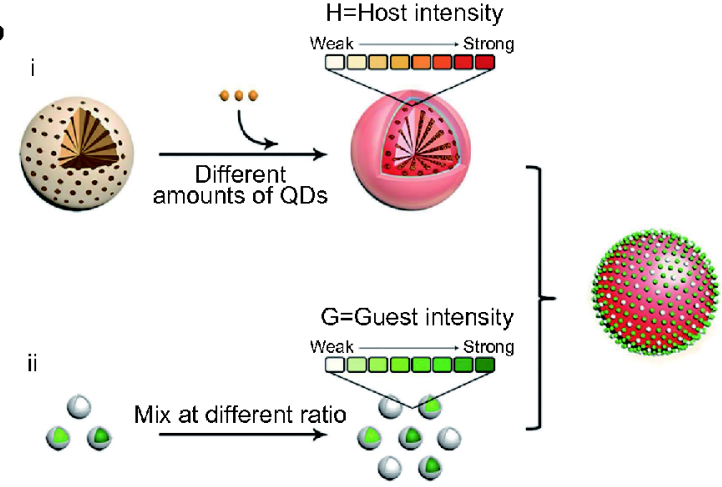

Figure 8 (a) Schematic of the fabrication process of QD-tagged PhC barcode particles with the combination of QDs and PhC barcodes. (b) Schematic of the hybrid-encoded barcode particle that possesses the strawberry-like structure in which large numbers of nanoparticles doped with dyes are tightly attached on the surface of QDs-encoded particles, resulting in outstanding capacity of encoding. (a) Reprinted with permission from Ref. [138]. Copyright 2014, the Royal Society of Chemistry. (b) Reprinted with permission from Ref. [140]. Copyright 2016, WILEY-VCH.

them with conventional methods. The analytes here are classified as ions, proteins, nucleic acids, and cells. And by means of rapid, high-throughput, multiplexed analysis of these analytes, we discuss the most promising applications of encoded barcode particles, such as disease diagnosis (cancer, heart disease, infectious disease), drug research and food and environment safety monitoring.

\section{Ions detection}

Health-related ions, including heavy metals (such as $\mathrm{Hg}^{2+}$, $\mathrm{Pb}^{2+}, \mathrm{Ag}^{+}, \mathrm{Cr}^{3+}$ ), electrolytes (such as $\mathrm{Na}^{+}$and $\mathrm{K}^{+}$) and others are closely connected with human life. The serious effects of heavy metal pollution on human health have made it a crucial environmental and health problem, for example, excess $\mathrm{Ag}^{+}$ions in the human body can result in a grayish-blue skin discoloration and $\mathrm{Hg}^{2+}$ ions can damage various organs and systems, such as kidneys, brain, immune system and other systems [142,143]. In addition, almost all higher forms of life need the electrolyte balance between intracellular and extracellular environment, and it is mainly dependent on the precise electrolyte gradients which can affect the blood $\mathrm{pH}$, nerve and muscle function and other body functions. Thus, if the concentrations of these important electrolytes change abnormally, serious health problem of the whole body may occur. There are also other ions that affect human health, for example, the abnormal concentration of $\mathrm{S}^{2-}$ can lead to liver damage or Alzheimer's disease. Thus, the monitoring of these health-related ions is crucial [144-146].

Several traditional approaches with wonderful sensitivity and specificity for detecting ions have been reported over the years, such as atomic absorption spectroscopy, voltammetric detection and inductively coupled plasma mass spectrometry [147-150]. However, these approaches usually need complex instruments, sophisticated sample preparation and other operations, whereas the multiplex test of metal ions has been increasingly crucial for environmental and health problems. Thus, it is required to develop an appropriate approach for simultaneous detection of different ions. Microarrays and various sensors have been made with regard to multiplex detection of ions [151]. For example, graphene oxide (GO) microarray and DNAzyme-based microarray both have been demonstrated a good method for multiplex determination of ions with satisfactory sensitivity and specificity. Various sensors including microfluidic device and other microsensor arrays also have been developed for multiplex ion detection [152-154]. However, the number of types of ions that can be detected simultaneously is usually limited. Thus, in order to realize high throughput detection of more kinds of ions, some barcode particles were then also developed for multiplex ion detection, including fluorescence-based, optically non-fluorescencebased and SERS-based ion sensing on the basis of detection method.

\section{Fluorescence-based ion sensing}

(i) DNAzyme/DNA molecules-functionalized ion sensing Recently, many studies have been carried out on DNAzymes. Because of their ability to select specific metal ions, DNAzymes are usually used to detect metal ions [155]. Moreover, the design of DNA molecules mainly depends on the capacity of the metal ions to form steady metal-DNA duplexes. Therefore, DNAzymes and DNA molecules have been converted into various sensors with some advantages of satisfactory specificity, stability and sensitivity for the potential application in ion detection [156-159].

Various DNAzymes-based sensors could be fabricated by binding pairs of fluorophore and quencher to different DNAzymes that are specific to metal ions such as $\mathrm{Ag}^{+}$, $\mathrm{Cu}^{2+}, \mathrm{Hg}^{2+}$ and so on $[160,161]$. Semiconductor QDs are a big change in the application of fluorophores for the 
fluorescence encoded barcode particles and multiplexed assays due to its outstanding advantages, so a novel QDDNAzymes-based sensor with high sensitivity and selectivity has been developed by combining the advantages of DNAzymes and QDs. In this study, the simultaneous multiplex detection of metal ions with a single excitation source could be realized in about half an hour [162]. As illustrated in Fig. 9a, in order to reach the highest possible quenching efficiency, both DNAzymes and substrates were bound with quenchers. When the target is absent, the quenchers bound to both DNAzymes and substrates on the QDs will lead to efficient quenching of the QDs. Whereas with the presence of the targets, the quenchers will keep away from the QDs along with the substrates and DNAzymes cleaving off from the QDs, resulting in the emission of QDs. The assay in this study could

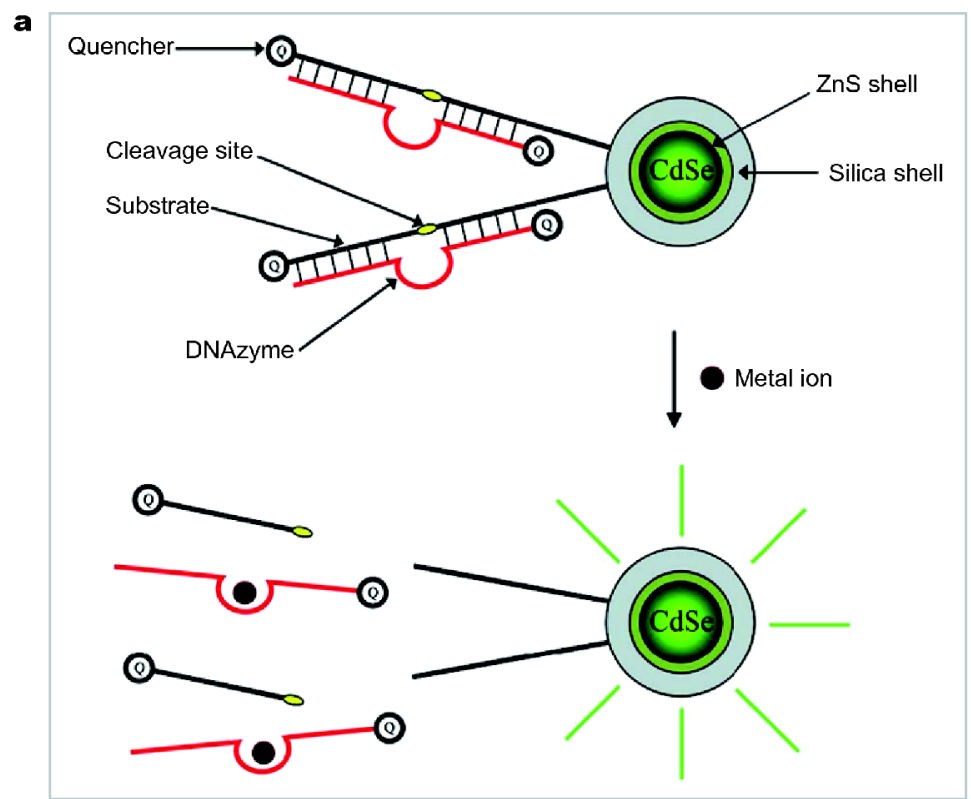

b

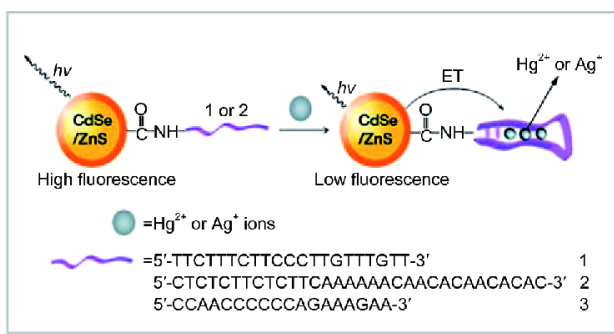

d
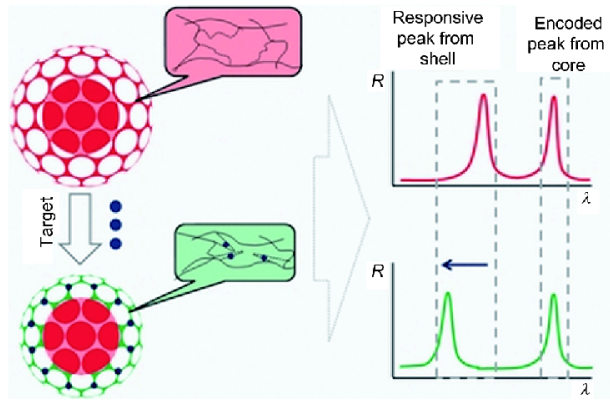

e

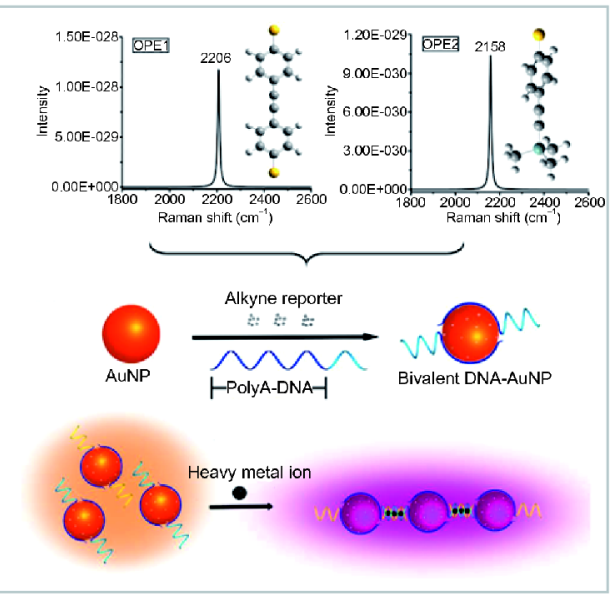

Figure 9 (a) Schematic of QD-DNAzymes-based sensor for metal ion detection. (b) Schematic diagram of the selective detection of $\mathrm{Hg}^{2+}$ or $\mathrm{Ag}^{+}$with $1-\mathrm{QD}_{560}$ or 2- $\mathrm{QD}_{620}$. (c) Fabrication of a multilayer sensor for $\mathrm{Na}^{+}, \mathrm{K}^{+}$and $\mathrm{H}^{+}$multiplex detection. Four different ion-sensitive fluorophores were employed into the cavity and three different kinds of QDs particles with different emissions were employed in the outer wall of the sensor. (d) Schematic of the PhC microcapsules with silica PhC encoding cores and responsive hydrogel PhC sensing shells for multiplex ion detection. (e) Density functional theory-calculated spectra of 4,4'-(ethyne-1,2-diyl)dibenzenethiol (OPE1) and 4-[(trimethylsilyl)-ethynyl]benzenethiol (OPE2) and schematic of SERS barcode particles with alkynyl group as Raman reporter for multiplex metal ion detection. (a) Reprinted with permission from Ref. [162]. Copyright 2010, American Chemical Society. (b) Reprinted with permission from Ref. [164]. Copyright 2009, WILEY-VCH. (c) Reprinted with permission from Ref. [165]. Copyright 2011, American Chemical Society. (d) Reprinted with permission from Ref. [108]. Copyright 2014, WILEYVCH. (e) Reprinted with permission from Ref. [168]. Copyright 2016, American Chemical Society. 
achieve the detection level of sub-nanomolar for different metal ions and it is more sensitive than other technologies at that time with about two orders of magnitude.

It is known that metal-DNA duplexes can also be obtained easily due to the high selectivity between DNA molecules and their corresponding type of metal ions. For example, stable $\mathrm{T}-\mathrm{Hg}^{2+}-\mathrm{T}$ or $\mathrm{C}-\mathrm{Ag}^{+}-\mathrm{C}$ duplexes can be obtained by binding $\mathrm{Hg}^{2+}$ or $\mathrm{Ag}^{+}$with their DNA molecules, and stable G-quadruplex can be obtained by binding $\mathrm{Pb}^{2+}$ with its unique DNA bases [163]. Therefore, by using QDs that are modified with T-rich or C-rich DNA molecules, $\mathrm{Hg}^{2+}$ and $\mathrm{Ag}^{+}$can be selectively analyzed with an electron-transfer-quenching path and the multiplex detection of metal ions can be realized by differentsized QDs [164]. Fig. 9b is the schematic diagram of the selective detection of $\mathrm{Hg}^{2+}$ or $\mathrm{Ag}^{+}$with $1-\mathrm{QD}_{560}$ or 2$\mathrm{QD}_{620}$, respectively. When $\mathrm{Hg}^{2+}$ or $\mathrm{Ag}^{+}$existed, the $\mathrm{T}$ or $\mathrm{C}$ residues of space-separated nucleotides could be linked with them to form a stable hairpin-like structure, and the finally formed $\mathrm{T}-\mathrm{Hg}^{2+}-\mathrm{T}$ or $\mathrm{C}-\mathrm{Ag}^{+}-\mathrm{C}$ duplexes then leaded to electron-transfer-quenching of the QDs. The results showed that the level of quenching was increased along with the increase of ion concentration and the limit of detection (LOD) was $2 \mathrm{ppb}$. In addition, a new kind of sensor for detecting different metal ions simultaneously has also been developed by combining PhC hydrogel microspheres with DNA molecules. When the target ions exist, the DNA-modified hydrogel could capture the targets specifically, resulting in the conformation of hairpin-like structure of DNA. Moreover, the green fluorescence could be observed as the sensing signals after adding SYBR Green I dye, and the reflection peaks of PhC microspheres could provide encoding signals.

(ii) Non-DNAzyme/DNA molecules-functionalized ion sensing

Besides heavy metal ions, a study for $\mathrm{Na}^{+}, \mathrm{K}^{+}$and $\mathrm{H}^{+}$ multiplex detection is presented here $[165,166]$. As shown in Fig. 9c, four different ion-sensitive fluorophores (FITC, SBFI, PBFI, and Dy647) were employed into the cavity of the microcapsules, and three different kinds of QDs particles with emissions of 577, 596, and $610 \mathrm{~nm}$ were employed in the outer wall of the microcapsules so that it is easy to encode different microcapsules ("001", "010", "011", "100", "101", "110", "111"). In this study, the ratio of blue-green to red from the different fluorescence in the cavity of the microcapsules was related with the concentration of $\mathrm{H}^{+}, \mathrm{Na}^{+}$, and $\mathrm{K}^{+}$, respectively, for example, FITC filled microcapsules could determine the concentration of $\mathrm{H}^{+}$, and SBFI or PBFI filled microcapsules could determine the concentration of $\mathrm{Na}^{+}$or $\mathrm{K}^{+}$, respec- tively. The results showed that it was feasible to measure the concentrations of different ions simultaneously by using such proposed sensor and it was possible to use it in cells.

\section{Optically non-fluorescence-based ion sensing}

The optical signals of responsive $\mathrm{PhC}$ can be changed when its refractive index or periodic lattice spacing changed, and it can be used as a good carrier for sensitive multiplex detection. A novel $\mathrm{PhC}$ microcapsules with silica $\mathrm{PhC}$ encoding cores and responsive hydrogel $\mathrm{PhC}$ sensing shells has been demonstrated for multiplex ion detection (Fig. 9d) [108]. In order to verify the sensing ability of such PhC microcapsules, the aptamers that were specific to $\mathrm{Hg}^{2+}$ or $\mathrm{Ag}^{+}$were employed to modify the hydrogel shells to fabricate different microcapsules. During the detection process, the targets would bind with their aptamers specifically to change the conformation of their aptamers. And these conformation changes could cause the shrinkage and the subsequent blue-shift of reflection peak of hydrogel shells, whose value can be assessed with target concentration.

\section{SERS-based ion sensing}

Besides the DNA-modified QDs sensor, DNA-modified SERS sensor is also a candidate for multiplex ion detection because of several advantages of SERS sensor. A novel AuNP-based SERS sensor has been demonstrated for detecting $\mathrm{Hg}^{2+}$ and $\mathrm{Ag}^{+}$simultaneously with the advantages of rapid response, high sensitivity and selectivity [167]. In this ion detection, three different kinds of thiolated DNA molecules were first employed to modify three same $20 \mathrm{~nm}$ AuNPs to constitute different conjugates of AuNP-DNA1, AuNP-DNA2 and AuNPDNA3, respectively. Then, aminothiophenol (ATP), nitrothiophenol (NTP) and 4-methoxy- $\alpha$-toluenethiol (MATT) were used as different Raman reporters to be attached on the three different DNA-modified AuNPs to display distinguishing SERS signals in the Raman scattering. When $\mathrm{Ag}^{+}$or $\mathrm{Hg}^{2+}$ existed, the AuNP-dimers could be formed through the $\mathrm{C}-\mathrm{Ag}^{+}-\mathrm{C}$ or $\mathrm{T}-\mathrm{Hg}^{2+}-\mathrm{T}$, resulting in the enhanced SERS signal from MATT or ATP because of the "hot-spot" between AuNP-dimers, while the AuNP trimers were formed when they coexisted, resulting in all higher enhancement of NTP, ATP, MATT SERS signals. Similarly, because the optical signal of alkynyl group is in the Raman silent region and there is no crosstalk between the silent region and visible region where most optical noise exists, so another SERS sensor for multiplex ion detection has been studied by using 
alkynyl group as Raman reporter (Fig. 9e) [168].

Environmental contaminants as a factor affecting human health are need to be monitored. Among them, heavy metal is one of the most common target. Thus, it is very important to detect various heavy metal ions in food resources rapidly and sensitively [169]. Such simultaneous detection of $\mathrm{Pb}^{2+}$ and $\mathrm{Cu}^{2+}$ in water by using DNAzyme-modified QDs or the simultaneous detection of $\mathrm{Ag}^{+}$and $\mathrm{Hg}^{+}$by using different T-rich or C-rich probes-modified barcode particles were all good examples of applications of barcodes in environment safety monitoring.

\section{Protein detection}

As an end product of gene expression, protein which serves to encode the information and acts as the working machinery of body to store and metabolize energy or regulate cell functions, is of great importance. They are assembled to different lengths by combining 20 kinds of different natural amino acids that are extended by the peptide linkage, resulting in their enormous structural diversity, giving rise to diverse functions of proteins. Based on their functions and responsibilities in living bodies, proteins can be divided into structural proteins or functional proteins $[170,171]$. The former refers to two forms of support materials such as tubulin in cellular cytoskeletons and collagen in connective tissue, while the latter has responsibilities to act as catalyser or transporter of small molecules. Thus, expressions of unique proteins can serve as biomarkers for activities in organisms or cells, while abnormal expressions of proteins often associate with pathological conditions, which can be used to diagnose specific diseases and for clinical research [172].

A lot of methods have been developed for the analytical techniques of protein detection, including physical, chemical and immunological methods. Physical method, such as fluorescence, Raman, ultraviolet (UV)/vis, mass spectroscopy (MS) or infrared radiation (IR) [173,174], and chemical methods are usually applied after an electrophoretic separation or chromatographic step, while the traditional immunological methods, such as the enzymelinked immunosorbent assays (ELISA) [175,176] and radioimmunoassays (RIA) do not need a separation step due to the accuracy and selectivity provided by the known antibody probes. However, these methods alone cannot be used to complete high-throughput multiplexed bioassays with the limited samples because they only detected a few targets separately at a time.

In order to bring along a suitable platform for highthroughput multiplexed assays, planar array (also called microarray), in which a good deal of reaction spots are arranged within a space of a few square centimeters, has become widely used for monitoring large numbers of proteins at a time with a very limited sample volume [177-179]. To date, numerous microarrays have been developed for protein analysis, such as DNA/RNA, whole protein, small molecule and peptide microarrays [180-182]. In addition, there are two groups of different detection methods in the usage of microarrays for the protein analysis, label detection method, such as fluorescent labeling, isotopic labeling, chemiluminescent labeling, electrochemically active probe labeling and nanoparticle labeling, and label-free detection method, such as MS, microcantilevers, quartz-crystal microbalance (QCM), surface plasmon resonance (SPR) and localized surface plasmon resonance (LSPR), and anomalous reflections of the gold surface (AR) [183]. In conclusion, label detection is the process to analyze the presence or activity of target by attaching extraneous molecules to the detection system chemically or temporarily, called labeling process. For example, fluorescent labeling and nanoparticle labeling generally require covalent bonding of coupled chemistry, and isotopic labeling analyzes the detection results by incorporating different elements into the targets. However, label detection usually needs complicated steps, long time and expensive instruments. Without these disadvantages of label detection, label-free detection that can monitor the inherent properties of the targets has been more popular. Instead of utilizing extraneous molecules, it can analyze the presence or activity of target through their inherent properties, such as molecular weight/charge and refractive index, and during the bioassays, reactions can be detected in the form of mechanical, electrical, or optical signals without labels. Thus, different high-throughput multiplexed assays can be realized by combining these detection methods with microarrays, while there are still some disadvantages in terms of the way that they are produced and used. Nowadays, due to its fast solution kinetics, large encoding capacity, rapid reaction time and rapid signal acquisition, barcode particles as microcarriers have been used in the multiplex protein detection by combining with different detection methods, such as fluorescence-based, SERSbased and label-free protein detection methods [184,185].

\section{Fluorescence-based protein sensing}

Immunoassay is the most widely used method for protein detection. It is a kind of biochemical test that shows the presence or concentration of an analyte in the complex solution by using a capture antibody (usually) which can 
completely distinguish the specific analyte from the mixture of different molecules. In addition, immunoassays usually employ a variety of different labels that can be chemically linked with the target antibody to allow for detection of analytes, among which fluorogenic reporter is one of the most popular labels. Thus, when fluorescence-based immunoassay is applied to barcode particle usage, several multiplex detection sensors have been developed. For example, permeable PEG hydrogel barcode particles have been used for multiplex protein immunoassay (Fig. 10a) [186]. It was composed of two separate regions. One region consists of some unpolymerized holes and is used as the graphical code, and the other region consists of one or more distinguishing antibody probe bands for multiplex target detection. This assay was a three-step sandwich immunoassay, including mixing barcode particles with the sample, adding biotinylated reporter antibodies and using the streptavidin- phycoerythrin (SAPE) complex to label the reporter antibodies. The results of the study showed that the LOD ranged from 1 to $10 \mathrm{pg} \mathrm{mL}^{-1}$, which is higher than that of traditional immunoassays. Moreover, another novel responsive hydrogel PhC particle is similarly used in multiplex immunoassay, which can realize signal amplification by shrinking their volumes to increase the fluorescence molecules density [187].

Besides organic fluorescent dyes, QDs-encoded nanoparticles as another fluorogenic reporter have also been used in barcode particle-based sandwich immunoassay [188]. As Fig. 10b illustrates, specific antibodies-modified QDs-encoded barcode particles (FEMMS) were used to detect targets, and QDs-encoded particles (QDs@SiO ${ }_{2}$ ) with antibodies were used as the reporters. The obvious red fluorescence signal could be observed on the barcode particles surface when the target was present, otherwise no fluorescence signals could be observed on the barcode
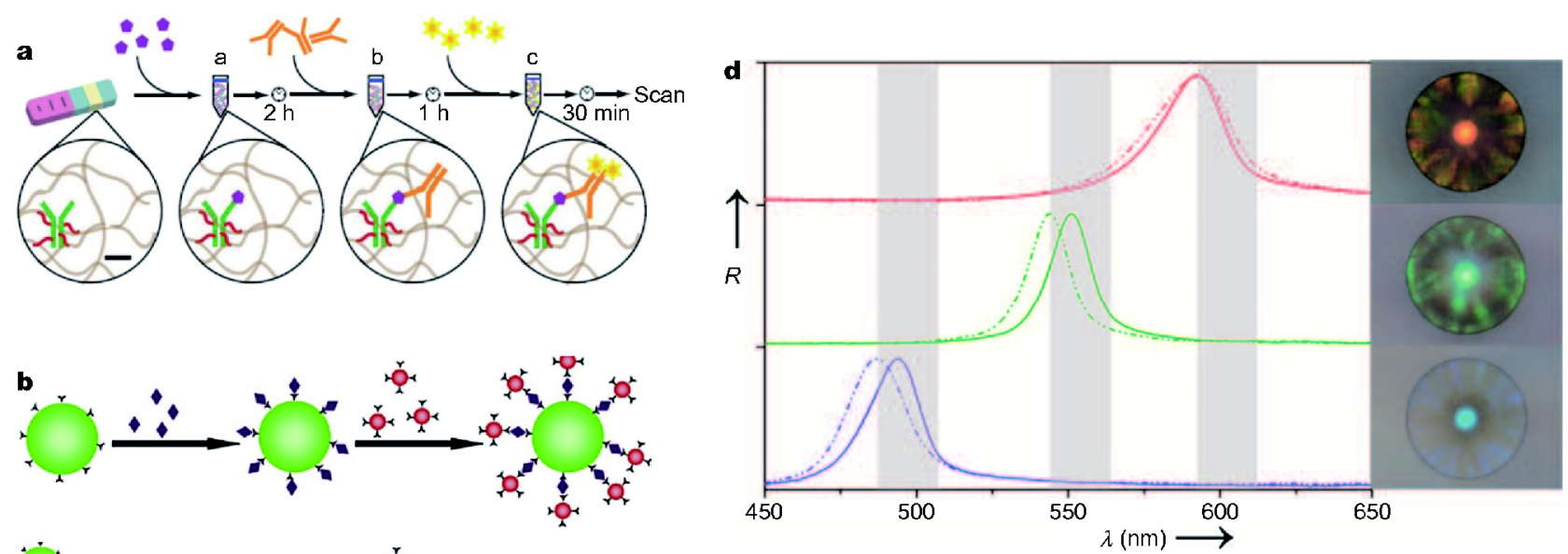

FEMMS-MMA II AFP

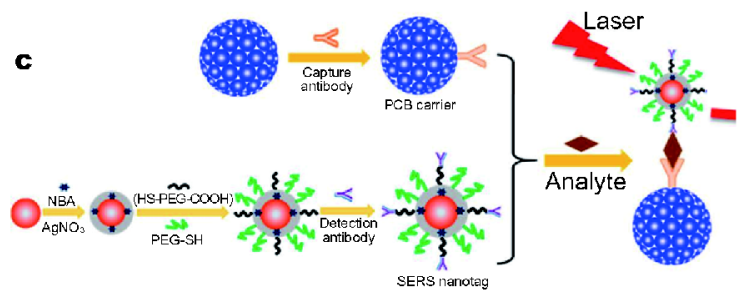

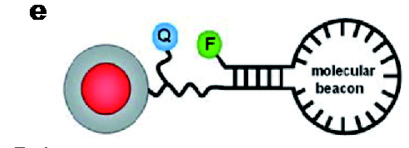

Polymer support off

Fluorophore Q Quencher

Figure 10 (a) Schematic of the protein immunoassay on permeable PEG hydrogel barcode particles. (b) Schematic of barcode particle-based sandwich immunoassay with QDs-encoded nanoparticles as fluorogenic reporter. (c) Schematic of protein immunoassay by using ultrasensitive biosensor with gold-silver core-shell SERS nanoparticles as labels and PhC barcode particles as encoding carriers. (d) Reflection spectra and optical microscopic images of three kinds of MIP barcode particles with different "footprints" of imprinted biomolecules. After protein detection, the diffraction peaks of the barcodes showed red-shift. (e) Schematic of label-free protein detection by immobilizing aptamer-based molecular beacon on the surface of fluorescence-encoded barcode particles. (a) Reprinted with permission from Ref. [186]. Copyright 2011, Nature America, Inc. (b) Reprinted with permission from Ref. [188]. Copyright 2016, Elsevier B.V. (c) Reprinted with permission from Ref. [189]. Copyright 2017, American Chemical Society. (d) Reprinted with permission from Ref. [191]. Copyright 2009, Wiley-VCH. (e) Reprinted with permission from Ref. [172]. Copyright 2012, MDPI. 
particles surface. Such results showed that the QDs-based immunoassay with good specificity could be employed for the successful applications.

\section{SERS-based protein sensing}

The amplified signals of SERS enhancement can be usually gained by using the Raman dyes as the labels and absorbing them on the noble metal particles such as gold or silver. An ultrasensitive biosensor has been proposed by using gold-silver core-shell SERS nanoparticles as labels and PhC barcode particles as encoding immunoassay carriers to detect protein with a wide range of concentrations [189]. As illustrated in Fig. 10c, after capture antibodies were immobilized on PhC barcode particles and combined with the targets specifically, Raman signal could be used as the detection results of the targets and the intensity of the measured signal was positively correlated with the target concentrations. It was also demonstrated that with high sensitivity and large encoding capacity, this sensor held promise for the multiplex protein detection. Moreover, as mentioned above, SERS nanotags can not only be used as the labels for immunoassays, at the same time also can be used together with $\mathrm{PhC}$ barcode particles as the dual encoding element, leading to larger encoding capacity.

There was another Ag-pyramid sensor in which SERS nanotags were used as both encoding carriers and labels [190]. Briefly, the Raman dyes-labeled AgNPs could be assembled by themselves to prepare Ag-pyramids by using "DNA-frame" that was embedded with aptamers specific to targets. Subsequently, in the presence of the analytes, the 3D-spatial geometries and the gap lengths of the pyramids could be altered due to the specific recognition between the analytes and the given aptamers, causing Raman signal enhancement. This assay realized the simultaneous, multiplex, and quantitative detection of proteins with the satisfactory efficiency and specificity by using Ag-pyramid biosensor, and the LOD was less than attomolar level.

\section{Label-free protein sensing}

(i) Molecular imprint-based protein sensing

Although certain labels are commonly used in immunoassays, some assays need no labels, but employ detection methods which need no modification and labeling. Molecularly imprinted polymers (MIPs) have the similar characteristics with the natural molecular receptors so that they can lead to unique applications in bioassays. However, traditional MIP biosensors are usually used for only single analyte detection. To over- come this situation, researchers have developed new types of MIP barcode particles to detect multiplex proteins with label-free methods instead of using traditional labeled immunoassays [191,192]. As shown in Fig. 10d, because of the coding characteristics of PhC, these MIP barcodes with the unique diffraction peaks had the capabilities for encoding, and by combining such capacity with the properties of molecular imprinting, these barcodes also could convert the invisible physicochemical changes to the visible optical signals. The specific explanation was that these MIP barcode particles had precisely amide groups and "footprints" of imprinted biomolecules, and when targets entered a complementary nanocavity, a large number of hydrogen bonds could be formed simultaneously between the amide groups and the targets. The results showed that the red-shift of the diffraction peaks of MIP barcodes could report the detection results of the targets, and shift position was positively correlated with the target concentration. Such detection results demonstrated that MIP barcode particles was the biosensor suitable for multiplex protein detection with good specificity and sensitivity, and as a novel type of barcode particles, their unique properties are anticipated to open new horizons in biodiagnostics [193].

\section{(ii) Molecular beacon-based protein sensing}

Molecular beacons have hairpin structures which can change their conformations spontaneously once hybridizing with their protein targets and then activate fluorescence resonance energy transfer (FRET). By immobilizing aptamer-based molecular beacon on the surface of fluorescence-encoded barcode particles, a novel sensor can be used for label-free multiplex protein detection (Fig. 10e) [172]. In this study, one side of the RNA aptamer which was specific to recognize target analytes was bound with a fluorophore, and the other side containing quencher was combined with the barcode particles. When the molecular beacons on the barcodes bound with the targets, the fluorophore of the molecular beacons could keep away from the quencher, resulting in the final fluorescent emission. Otherwise, the barcode particles showed only one color at the core layer. Thus, the targets can be easily recognized by the fluorescence emission of molecular beacons without any further labeling step.

\section{Practical application}

As one of the most important form of biomarkers, these protein detection methods were employed for disease diagnosis, such as cancer, heart disease, Down's syndrome and so on. Cancer is currently one of the most lethal diseases in the world, and it usually derives from 
the growth of abnormal cells with the disease progression of invading and spreading to the rest of body regions. Most of cancers are usually found according to the obvious symptoms or by the screening. However, people usually have terminal cancers when they are diagnosed because of lack of the incipient symptoms. Unlike the diagnosis that arises from symptoms or signs of body, early cancer screening is usually performed after cancer has existed, whereas without the appearance of obvious symptoms. The screening methods include body fluid tests, imageological diagnosis and so on. Among them protein tumor biomarkers in blood, urine or other body fluid generated by either tumor cells or non-tumor cells when the tumor has existed can be used to screen and evaluate cancer diseases early and monitor the ther- apeutic effect of cancer. However, because of their limited specificity and sensitivity resulting in false positive and negative tests, multiplex protein tumor biomarker test is needed. For example, an effective multiplexed bioassay for detecting multiple lung cancer-related biomarkers from a single sample simultaneously was developed by combining magnetic beads and QDs (Fig. 11a) [194]. The bioassay was carried out with the sandwich method in which the capture probes were linked onto the magnetic beads, and meanwhile the second antibodies were linked onto QDs with different emissions for different targets. The results of the multiplex reactions showed that the three different immune-complexes could easily distinguish the three targets and their fluorescence intensities were all positively correlative with the concentrations of $\mathbf{a}$
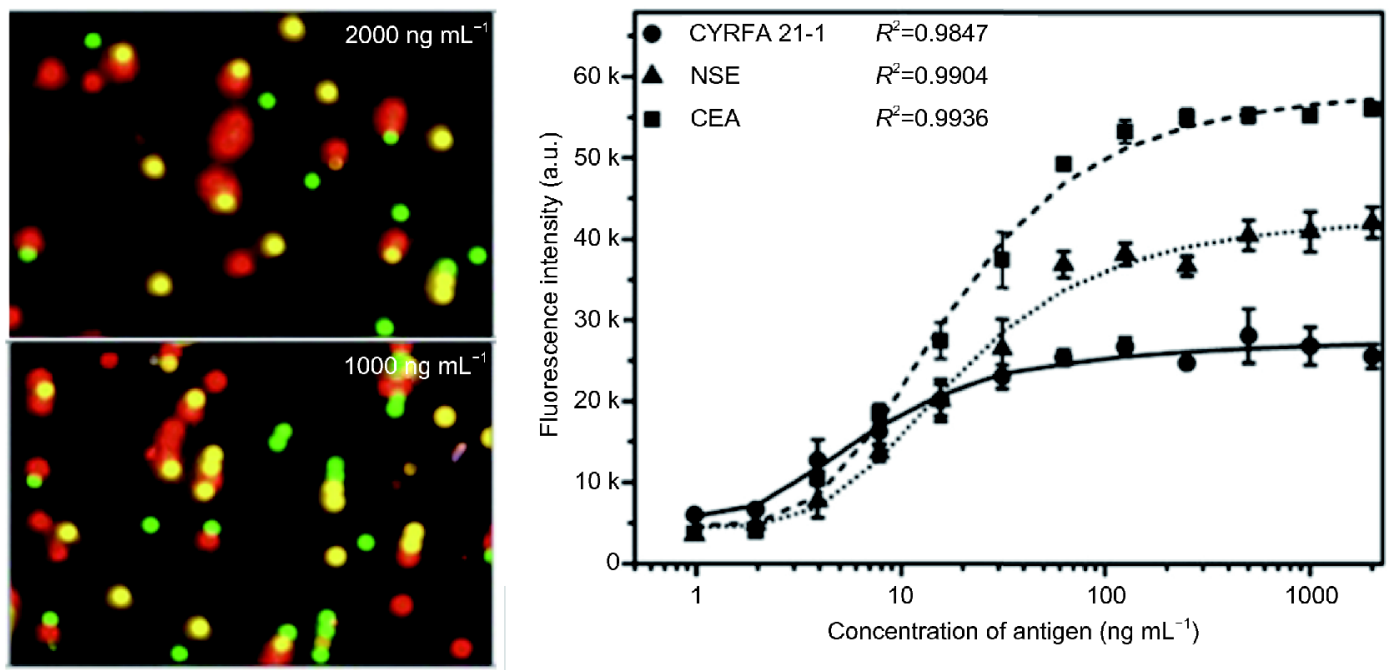

c

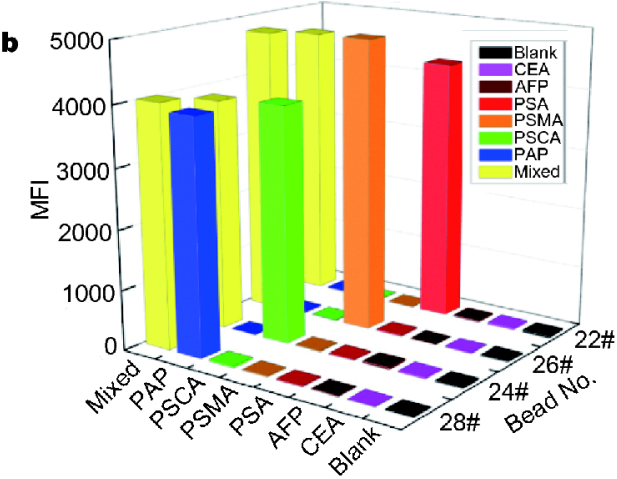

c 120

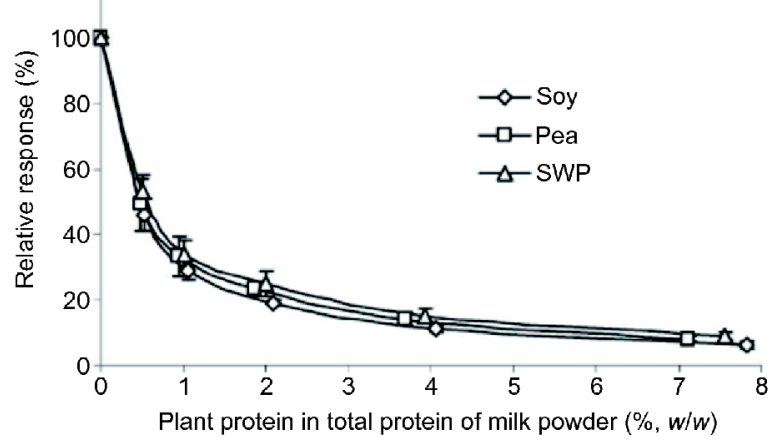

Figure 11 (a) Fluorescence graphs of the detection results of different concentrations of CYRFA21-1, NSE and CEA and the standard curves for these three biomarkers in the multiplex bioassay. (b) The assay results of the reactions between the mixed four coupled barcodes and different targets. (c) Calibration curves of the multiplex plant proteins detection in milk powder. (a, b) Reprinted with permission from Ref. [194,196]. Copyright 2016 and 2013, Elsevier B.V. (c) Reprinted with permission from Ref. [200]. Copyright 2007, American Chemical Society. 
these three targets. Moreover, the LOD was $364 \mathrm{pg} \mathrm{mL}^{-1}$ for CYRFA21-1, $38 \mathrm{pg} \mathrm{mL}^{-1}$ for CEA, and $370 \mathrm{pg} \mathrm{mL}^{-1}$ for NSE, and it could completely satisfy clinical requirements. Besides these three protein biomarkers, $\alpha-1-m i-$ croglobulin/bikunin precursor (AMBP), peroxiredoxin 2 (PRDX2), and Parkinson disease protein 7 (PARK7) as additional lung cancer-related biomarkers were simultaneously detected with the QD-encoded barcodes-based sandwich immunoassay [195]. This study focused on the comparison of the results obtained in the QD-encoded barcodes-based sandwich immunoassay and the alternative Luminex xMAP bead-based immunoassay and the data showed that this QD-based technology may be considered an alternative to Luminex xMAP technology for the diagnosis of cancer diseases.

Similarly, the protein tumor biomarkers also play an important role for prostate cancer screening and diagnosis, which is the third most common cancer in men. For prostate tumor screening, prostate-specific antigen (PSA), prostatic acid phosphatase (PAP), prostate-specific membrane antigen (PSMA) and prostate stem cell antigen (PSCA) were simultaneously detected by using fluorescent dyes-encoded barcode particles [196]. Unlike the common sandwich immunoassay, this assay was performed in the format of competition between the targets bound on the barcodes and the free targets for their corresponding antibodies in solution. As shown in Fig. $11 \mathrm{~b}$, no matter in single or multiplex tests, the protein coupled barcodes only reacted with the matching targets and the results of median fluorescence intensity (MFI) was from 3,834 to 4,832, meanwhile the signals in the control groups showed no non-complementary reactions with the MFI less than 50. These results indicated the favorable specificity for multiplexed detection of prostate cancer biomarkers, and with the LOD of

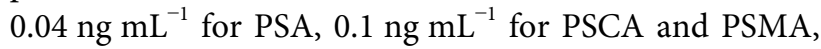
$0.173 \mathrm{ng} \mathrm{mL}^{-1}$ for PAP, this technology was applicable for extensive screening of prostate cancer in high-risk population.

Acute myocardial infarction (AMI), as an important health problem, is the primary cause of irreversible heart failure (HF) and the death that followed every year. However, coronary heart disease (CAD) is the main cause of AMI, suggesting the great importance of early diagnosis of CAD to improve survival rates significantly by offering prime-time medical treatment. C-reactive protein (CRP), cardiac troponin I (cTnI) and B-type natriuretic peptide (BNP) can collectively serve as protein biomarkers of CAD. Thus, multiplex detection of these three biomarkers based on the $\mathrm{PhC}$ barcode particles has been developed to realize the simple diagnosis of CAD [197]. The results showed that cTnI, CRP and BNP could be detected with the LOD of $0.01 \mathrm{ng} \mathrm{mL}^{-1}, 0.36 \mathrm{mg} \mathrm{L}^{-1}$ and $0.004 \mathrm{ng} \mathrm{mL}^{-1}$, respectively. And the results of the clinical application showed no significant difference with that of commercially proven ECLIA method, demonstrating the good analytical reliability. Based on the recent study, myoglobin level usually has already been elevated when the test results of cTnI stayed in its normal range for the patients with myocardial infarction, suggesting that the multiplex detection of cTnI and myoglobin could improve the sensitivity of diagnosis. This multiplex assay has been realized by gold nanorod [198].

Down's syndrome, also named as trisomy 21 , is caused by the third copy of chromosome 21 in fetus. This disease can lead to some problems, such as typical faces and a certain degree of intellectual retardation. Thus, it is very important to avoid this disease by detecting the biomarkers for Down's syndrome in a specific period of pregnancy. Among the biomarkers, alpha fetoprotein (AFP) and free Beta human chorionic gonadotropin ( $\beta$ hCG) are commonly detected during about 20-week pregnancy to assess the risk of this disease. In order to reduce sample volumes and reaction time, these two biomarkers have been detected simultaneously on $\mathrm{PhC}$ barcode particles [199]. The results showed that there was positive correlation between the fluorescence intensities and target concentrations, ranging from 1 to $1,000 \mathrm{ng} \mathrm{mL}^{-1}$ that were enough for practical application and because the cross-reactivity between these two targets could be ignored, this method could be employed for multiplexed detection. And the acceptable accuracy and detection reproducibility suggested that this barcode particle is potential for screening the risk of Down's syndrome.

In addition, as one of the factor of food safety, food adulteration could be monitored by detecting corresponding adulterants. For example, some low-cost vegetable proteins have become the common adulterants for producing milk powder or other dairy products. A triplex study demonstrated a reliable approach to detect and quantify the non-milk proteins in the dairy products by combining xMAP technology with competitive immunoassay (Fig. 11c) [200]. In this study, soluble pea proteins, wheat proteins and soy proteins were coupled with three different barcode particles of different colors and the sensitivity of this study was verified as about $0.5 \mu \mathrm{g} \mathrm{mL}^{-1}$ at $50 \%$ binding.

\section{Nucleic acids screening}

Besides proteins, nucleic acids as one kind of the most 
important bio-macromolecules in the living organisms, can encode and store unique genetic information in the nucleus of different living cells. The name nucleic acid is the generic term for deoxyribonucleic acid (DNA) and several types of ribonucleic acid (RNA). They are typical linear bio-macromolecules that consist of three kinds of different subunits, among which 5-carbon sugars and phosphates can be combined to form the backbones of the nucleic acids, and meanwhile the nucleobases with different numbers and specific orders can preserve and transmit their unique genetic information [201]. After the accomplishment of Human Genome Project and the great progress of nucleic acids synthetic technologies, nucleic acids have been given much attention both in basic research and clinical applications, such as accurately identifying their sequences and reproducibly quantifying their expression levels.

The physician in clinics usually uses an 'exclusion' method to diagnose diseases, but it usually costs too long time to get the right diagnosis and the following efficient treatment, whereas the multiplexed bioassays that follow the 'inclusion' method can draw an accurate diagnosis and give efficient treatment in a short time [202]. Thus, the disability of traditional nucleic acids analysis approaches including northern blot, polymerase chain reaction (PCR) and quantitative PCR (qPCR) to realize high throughput multiplex detection largely limit their applications. There are diverse technologies to accomplish multiplexed nucleic acid assays, one of which is about assay chemistry, such as multiplex PCR (the most commonly used) [203,204], multiplex RT-PCR [205,206], multiplex minisequencing (primarily applied to SNP detection) [207], multiplex isothermal amplification (applied to RNA detection) [208], multiplex oligonucleotide ligation-PCR [209] and so on. Another technology is about detection platform, such as solid-phase microarrays and suspension arrays [210-213]. The genomic hybridization is commonly used on solid-phase microarrays, and in such assays the microarrays are usually modified with the oligonucleotides that are specific to target nucleic acids and these target nucleic acids labeled with fluorochrome are then hybridized with the microarray. This solid-phase microarray related bioassay without any amplification strategy has been widely and successfully used. Meanwhile, with its unique advantages, suspension arrays based on barcode particles have also been developed for high throughput multiplex nucleic acid detection, among which are fluorescence-based, SERS-based and label-free nucleic acid sensing on the basis of the detection method.

\section{Fluorescence-based nucleic acid sensing}

Similar as other technologies, the detection of nucleic acids on barcode particles is also performed based on nucleic acid hybridization between the probe and target sequences complementary to each other, among which sandwich hybridization assay with fluorescent labeling is the most commonly used. According to the various reflectivity of neighbouring metal stripes, the sub-micron metallic nanowires can be used as the coding carriers for SNP detection [214]. As illustrated in Fig. 12a, each kind of nanowire was firstly conjugated with a unique oligonucleotide probe and another oligonucleotide probe labelled with fluorescent dye and the targets were then added into the detection system. In this study, these two kinds of probes both could hybridize with the same target specifically, so when the target SNP existed, they could ligate together after the hybridization and lead to fluorescence signals. In Fig. 12a, two kinds of probes were ligated when Allele A was complementary to the target SNP, whereas the ligation did not happen when Allele G was mismatched to the target. Another sandwich hybridization assay with fluorescent labeling has also been reported with the use of QDs barcode particles to detect three genes of Amp, Neo, and Lac Z simultaneously [215]. The results showed that short probes and short target nucleic acid could produce best hybridizing rate and the LOD was $0.02 \mathrm{fmol}$.

In addition, the development of assays to quantify multiplex microRNA simultaneously with graphically encoded barcode particles and PhC barcode particles has been described [216,217]. As demonstrated in Fig. 12b, different probes were firstly fixed on the "probe" regions of the barcode particles, and after adding these barcodes into the reaction system, the target microRNAs could be captured by the barcodes that modified with the targetspecific probes, and finally the target microRNAs captured by the probes were labeled with fluorescent dyes to show the appearance and the quantification of the target. Moreover, this study also realized the signal amplification by employing a universal adapter to carry out the rolling circle amplification (RCA) approach during the detection process. With the use of RCA approach to amplify the fluorescent signals, such barcode particles with the great sensitivity could detect trace amounts of microRNAs directly from the blood sample and needed neither target extraction nor target amplification approaches. Thus, in comparison with other arrays and traditional RCA techniques, these barcodes with both superior sensitivity and the ability of multiplex detection have a wide range of applications. 

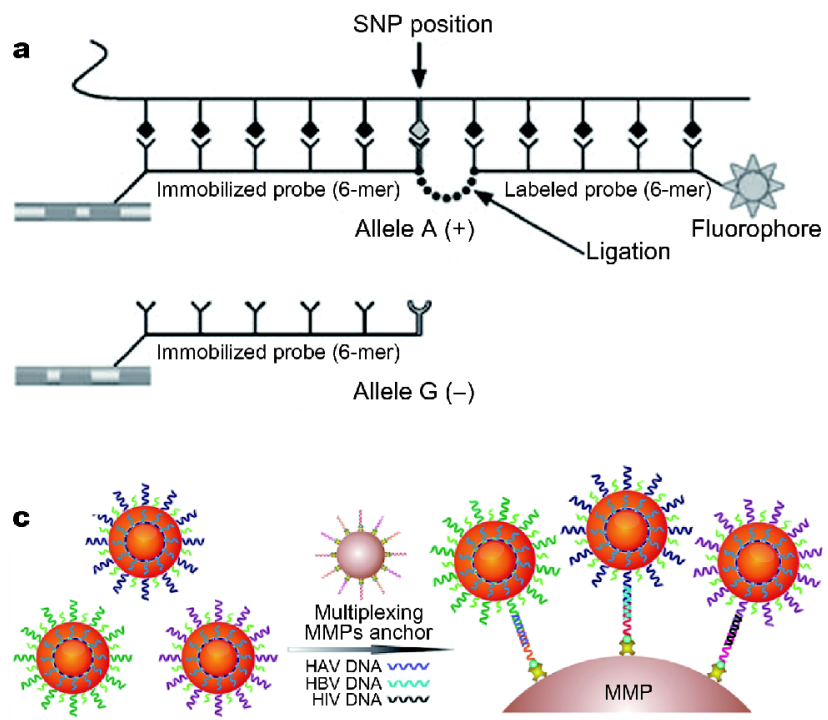

$\mathbf{e}$
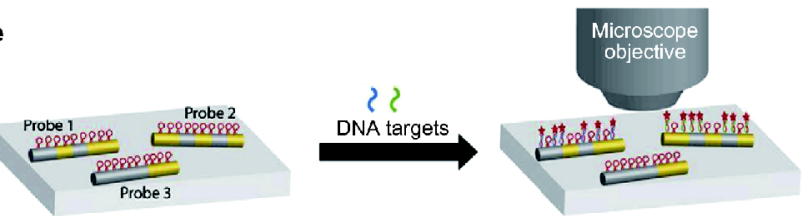

b
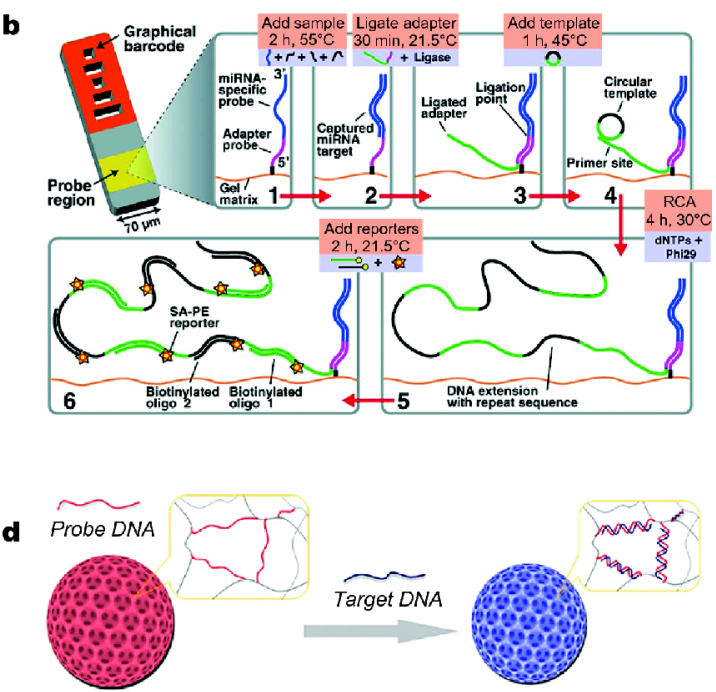

$\mathbf{f}$

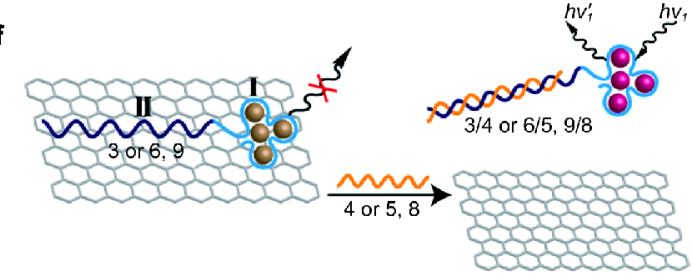

Figure 12 (a) Schematic of SNP detection by using sub-micron metallic nanowires as the coding carriers. (b) Schematic of multiplex microRNA detection with graphically encoded barcode particles and RCA strategy. (c) Schematic of multiplex gene quantitative detections by using different SERS nanoparticles that were modified with different probes. The target nucleic acids could be bound with the corresponding SERS nanoparticles because of the specific recognition between the targets and DNA probes. (d) Schematic of multiplex DNAs detection without labelling on the DNAresponsive hydrogel PhC barcode particles. (e) The process of multiplex label-free gene detection with metallic nanowires that were modified with molecular beacon probes as encoded barcodes. (f) Schematic of label-free DNA detection using fluorescent DNA sensor consisted of nucleic-acidmodified AgNCs and GO. (a) Reprinted with permission from Ref. [214]. Copyright 2006, Springer-Verlag. (b) Reprinted with permission from Ref. [216]. Copyright 2011, American Chemical Society. (c) Reprinted with permission from Ref. [218]. Copyright 2014, the Royal Society of Chemistry. (d) Reprinted with permission from Ref. [220]. Copyright 2010, WILEY-VCH. (e) Reprinted with permission from Ref. [221]. Copyright 2008, Elsevier Ltd. (f) Reprinted with permission from Ref. [222]. Copyright 2013, American Chemical Society.

\section{SERS-based nucleic acid sensing}

Besides fluorescent labeled sandwich immunoassay, SERS encoded particles, with the advantages of unique structure, rapid detection speed, great sensitivity and strong operability in complicated reaction, also have been widely used for multiplex detection of nucleic acids. For example, by using stable SERS signal as the nanoprobe, novel core-shell gold nanoparticles were synthesized for multiplex gene quantitative detections (Fig. 12c) [218]. Three SERS nanoparticles encoded by different SERS reporters were functionalized with different DNA probes that were specific to HBV, HIV and HAV, respectively. And because of the specific recognition between the targets and DNA probes, the target nucleic acids could be bound with the corresponding SERS nanoparticles to form sandwich complexes. The results showed that the unique SERS peaks could be observed only when these corresponding targets existed in the sample, and the intensities of these SERS peaks increased with the increasing target concentrations. A similar multiplex nucleic acid assay using SERS nanoparticles as probes was also reported to simultaneously detect two kinds of DNA markers (Kaposi's sarcoma-associated herpesvirus and bacillary angiomatosis) [219].

\section{Label-free nucleic acid sensing}

(i) Spectral signal-based nucleic acid sensing

Most of the suspension arrays for multiplexed nucleic acid detection are usually labelling immunoassay methods, but these methods commonly need complicated steps, long time and expensive instruments. Thus, labelfree detection is in demand due to its simple report 
strategy. One kind of DNA-responsive hydrogel $\mathrm{PhC}$ barcode particles has also been developed to detect multiplex DNAs without labelling (Fig. 12d) [220]. Similar to optically non-fluorescence-based ion sensing and molecular imprint-based protein sensing, the hydrogel photonic barcodes also could change the invisible physicochemical signals into visible optical signals. When target DNAs contacted with the probe DNA-modified barcodes, they would be hybridized with the probe DNAs on the barcodes specifically, causing the hydrogel to shrink. Finally, the visible change of the optical signals could be estimated by blue-shift diffraction peaks of the barcode particles and the degree of the change was related with the amount of target DNAs. This technology could become a low-cost, simple and sensitive platform to realize multiplex nucleic acid detection.

(ii) Molecular beacon-based nucleic acid sensing

In addition to the fluorescence-based bioassays, metallic nanowires are also used for label-free detection with molecular beacon [221]. As illustrated in Fig. 12e, different nanowires with unique patterns of $\mathrm{Au}$ and $\mathrm{Ag}$ segments were modified with unique molecular beacon probes, respectively. When the target nucleic acids existed, and reacted with the nanowires, the molecular beacon probes bound to the nanowires would hybridize with them specifically and become fluorescent. On the other hand, the molecular beacon probes would keep non-fluorescent when the target nucleic acids were absent. After the detection, the reflectance images of the nanowires were used to identify the types of the probes and the fluorescence images were used to quantify the fluorescence intensities which were related with the amount of target nucleic acids.

\section{Fluorescence-based nucleic acid sensing}

Unlike the fluorescent labeled immunoassays, this kind of fluorescence-based bioassay is label-free [222]. As illustrated in Fig. 12f, this fluorescent DNA sensor consisted of nucleic-acid-modified silver nanoclusters (AgNCs) and graphene oxide (GO). In the multiplex detection, two types of AgNCs were used for encoding, including the red-emitting AgNCs $(616 \mathrm{~nm})$ and nearinfrared-emitting AgNCs $(775 \mathrm{~nm})$. Without target DNAs, the singlestranded DNA probes were conjugated with the nucleicacid-modified AgNCs and they were both adsorbed to $\mathrm{GO}$, resulting in the quenching of AgNCs. Whereas, when target DNAs existed, they would hybridize with the single-stranded probes and formed double-stranded DNAs to make the AgNCs desorb from GO, along with the visible fluorescence signals. This label-free fluorescent sensor also can be used for detecting other molecules, such as ATP and thrombin.

\section{Thermophysics-based nucleic acid sensing}

The solid to liquid phase change materials (PCM) have unique thermophysical properties. PCM could be melted by adsorbing heat energy without temperature change. Thus, the phase change metal nanoparticles that were encapsulated with silica were used as a novel kind of barcode particles. In this research, by using various types of materials, each type of target had its unique relationship with the melting temperature of barcodes [223]. In addition, because of the higher melting temperature of $1,650^{\circ} \mathrm{C}$, the silica shells which were modified with the single strand DNA probes would not melt, and the detection signal could be read out with differential scanning calorimetry, where the melting temperature and the latent heats of fusion were used to show the existence and concentration of the target.

\section{Practical application}

As another important form of tumor biomarkers, these nucleic acid detection methods have also been employed for cancer diagnosis. During cancer development, there are strong correlations between certain types of abnormal microRNAs and tumorigenesis, even other developing process of cancer such as invasion and metastasis. Let-7 is one of the popular tumor suppressor microRNAs, and the downregulation of its family members can usually be observed in many cancer types, for example, a general downregulation of let-7 was found in lung cancer, let-7d was found in ovarian cancer and head and neck squamous cell cancer, let-7e and let-7i were found in prostate cancer, and so on [224]. A graphically encoded barcode particles has been reported for multiplexed detection of four let-7 family members (let-7a, b, d, f) [225]. With the estimated detection limit of $5 \mathrm{amol}$ and the advantages of less cost and assay time, this technology offers unlimited prospects for clinical applications. In addition, similar graphically encoded barcode particles used for multiplex detection of other cancer-related microRNAs (microRNA-141, microRNA-210 and microRNA-221) confirmed the correlations between the upregulated microRNA-141 and prostate cancer (Fig. 13a) [216]. Thus, the multiplex microRNAs detection with barcode particles could be helpful for creating new method to realize noninvasive cancer diagnosis.

Multiplex gene detection methods also could be employed for infectious disease monitoring. The rapid and effective real-time monitoring can halt further travel of 

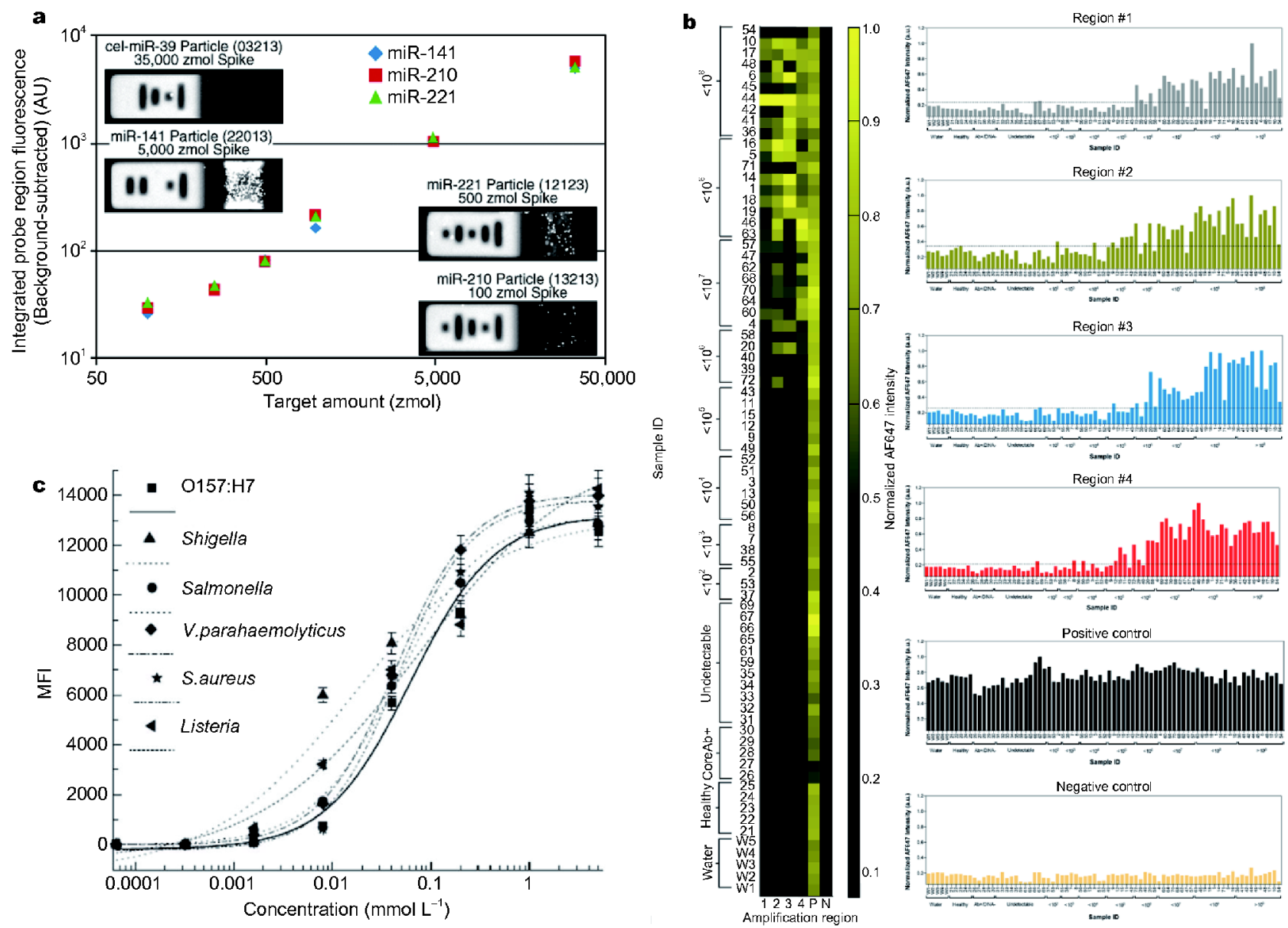

Figure 13 (a) Calibration curve for multiplex miRNAs detection, and the cel-miR-39 particles were used as the control group. (b) Heat map diagram and bar graphs of multiplex detection of four amplification regions. (c) Standard curves of multiplex foodborne pathogen detection results with barcode particles. (a, b) Reprinted with permission from Ref. [216,227]. Copyright 2011 and 2016, American Chemical Society. (c) Reprinted with permission from Ref. [21]. Copyright 2013, Elsevier Ltd.

the diseases because patients can be quarantined or get proper treatment for the disease after being diagnosed [226]. Currently, there are several common infectious diseases that can cause high mortality in the world, such as syphilis, HIV, malaria, hepatitis B, and hepatitis C. Thanks to the development of molecule detection, these infectious diseases can be diagnosed or distinguished successfully by detecting gene fragments simultaneously from the genome of these diseases based on barcode particles. For example, several gene fragments of hepatitis $B$ virus (HBV) which causes more than 0.5 million deaths every year, have been detected by using QDs barcode particles with improved clinical sensitivity of $80.4 \%-$ 90.5\% [227]. As demonstrated in Fig. 13b, the signals of the barcode particles in positive groups were more than 3 -fold higher than that from the barcode particles in negative groups, confirming that this 6-plexed test could work correctly. In addition, In comparison with other fragments from the same HBV gene, some fragments could produce signals of higher intensity, indicating the necessity of multiplexed bioassays to improve the accuracy by reducing false negative rates and increasing true positive rates. Besides hepatitis $\mathrm{B}$, influenza, HIV and hepatitis $\mathrm{C}$ are also diagnosed by detecting their gene fragments on QDs barcode particles [228]. One transportable smartphone also has been developed as diagnostic device, which was easy to use and enabled the radio transfer of diagnosis for interpretation to realize the realtime monitoring and prediction of diseases [229].

Pathogen, as another important factor affecting food safety, is a serious threat to human health. It is necessary to detect pathogens rapidly for stopping their outbreaks in the mass populations. Several suspension arrays have been studied to realize multiplex gene detection of 
foodborne pathogens. For example, specific oligonucleotide probes-modified fluorescent encoded barcode particles have been reported to simultaneously detect the specific genes of Escherichia coli O157:H7 (E.coli), Shigella, Salmonella, Vibrio parahaemolyticus, Staphylococcus aureus and Listeria monocytogenes [21]. The results showed that the six probes involved no cross-reaction and the median fluorescent intensity of multiple testing was higher when the concentration of pathogens increase with the detection limit of $1.6 \times 10^{-6} \mathrm{mmol} \mathrm{L}^{-1}$ (Fig. 13c).

\section{Cell assay}

Cell is the basic composition of all organisms. The human bodies consist of countless cells to form the body structures. Moreover, cells have their specialized duties, such as taking nutrients from foods and then converting them into energies. Cells also carry genetic materials and can copy them forever. Moreover, in all living organisms, there are various types of cells that have different functions. And besides the normal cells in body, there are abnormal cells undergoing uncontrolled growth, called cancer cells, which can be induced by both internal factors including gene mutation, hormones and immune condition, and external factors such as chemical and infectious agents and radiation. Thus, due to the important role of cells, more and more studies have been developed for learning more about cells, including nanostructures and biomolecular (such as protein and genomics) processes of cells, capture and detection of cancer cells, cultivation of cells for other studies and so on.

\section{Cell imaging}

To study nanostructures and biomolecular processes of cells under normal and diseased conditions, nano-barcodes, with their nano-size feature, have been prevalently used as tags for biomolecule labelling, especially in cell imaging [230-232]. For cell imaging purposes, the nanobarcodes should be functionalized with an additional layer of biocompatible material and the toxicity of nanobarcodes is also an important consideration. Currently, fluorescent encoded barcodes which can be observed easily under a fluorescence microscope are the most commonly used as labels for cell imaging. For example, QD encoded barcodes modified with cyclic Arg-Gly-Asp (cRGD) targeting peptides were demonstrated for uptake by human breast cancer and cervical cancer cells (Fig. 14a) [233]. The QDs encoded barcodes have the advantages of strong optical signals and stable chemical properties, so they could be used for in vitro and in vivo imaging. The results showed that the cRGD-modified
QDs encoded barcodes mainly targeted the $\alpha_{v} \beta_{3}$-high expressing MCF-7 breast cancer cells instead of the $\alpha_{v} \beta_{3}$ low expressing HeLa cervix cancer cells. It was indicated that the cRGD targeting peptides could promote the uptake of the barcodes by those $\alpha_{v} \beta_{3}$-expressing cells and this feature of the barcodes could offer them wide applications in noninvasive nano-imaging and nano-diagnosis. As another kind of fluorescent barcodes, the semiconducting polymer dots (Pdot) that could be excited by only single wave also have been developed to track single cells [234]. These barcodes were used to tag 20 types of cancer cells and finally these cancer cells could be identified by using flow cytometry. The results suggested that Pdot barcode particles had the great ability to discriminate various cells and track them in a long time.

In comparison with fluorescent encoded barcodes, Raman encoded barcodes have the benefits of nil autofluorescence (for down-converting fluorescent particles), little spectral overlap and absence of photobleaching, and thus SERS encoded barcodes are also commonly used as labels for cell imaging. Earlier, multiplexed in vivo imaging in the liver of living mice was performed by using SERS barcodes as can be seen in Fig. 14b [235]. The results showed that there were ten different SERS barcodes in the same living mouse after intravenous injection. Thus, because of the ability of multiplex of ultrasensitive SERS barcodes, it is possible to target more molecules simultaneously in vivo to realize simple noninvasive disease screening. Moreover, SERS encoded hollow barcodes conjugated with antibodies on the surface were used for multiplex imaging of the epidermal growth factor (EGF), ErbB2, and insulin-like growth factor-1(IGF-1) for three human breast cancer cell lines MDA-MB-468, KPL4 and SK-BR-3 and another SERS encoded barcodes have also demonstrated the multiplex imaging of five different types of breast cancer cells present in a cell coculture $[236,237]$.

\section{Cell molecular analysis}

Most of the pathology of living systems is usually reflected by the genetic variation in the cells, and the gene expression whose variation contributes to disease risk could be understood through the changes in different levels, such as RNA levels, protein levels, and so on. Thus, the biomolecules in the cells not only can be used for cell imaging, but also can be used to analyze the gene expression for disease detection. The droplet microfluidics has been exploited to develop a technology to index large amounts of cells and sequence their RNAs [238,239]. As can be seen in Fig. 14c, the novel device of microfluidics 
a
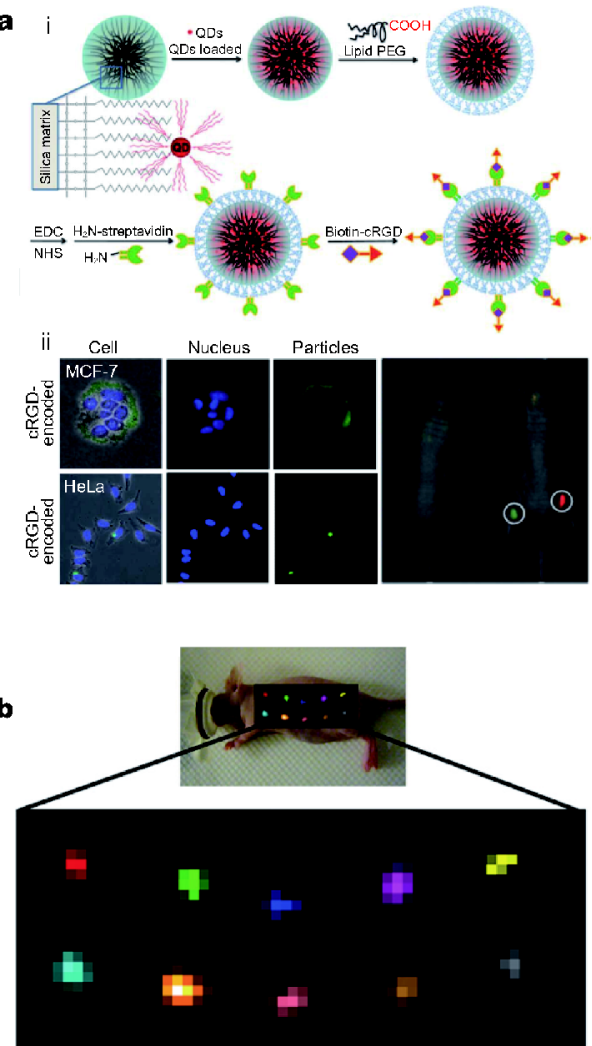

c

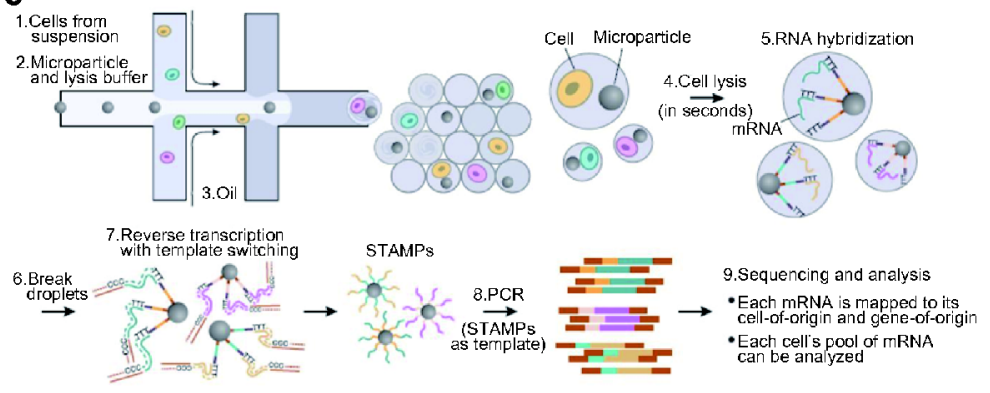

d
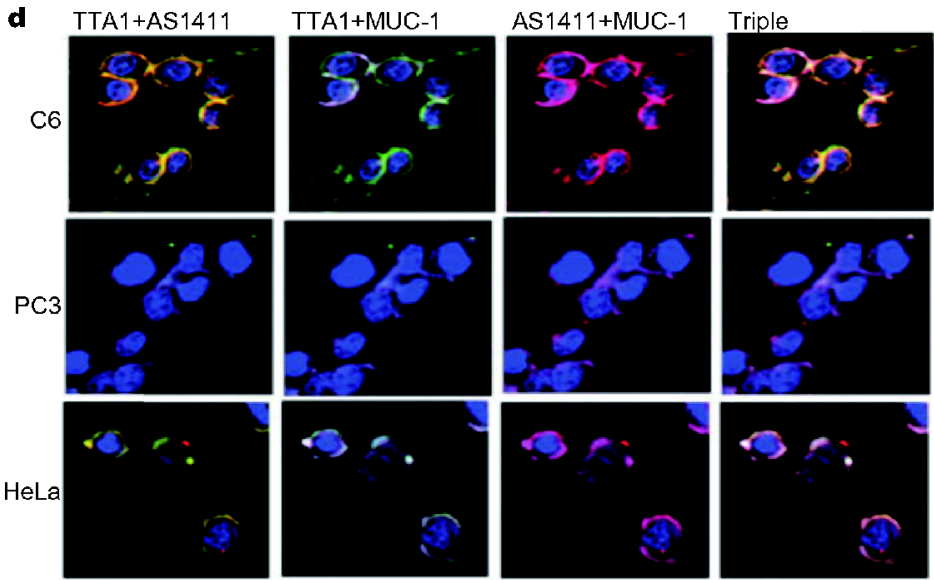

Figure 14 (a) QDs encoded barcode particles were used as labels for cell imaging: (i) different QDs encoded barcode particles modified with cyclic Arg-Gly-Asp (cRGD) targeting peptides, (ii) in vitro and in vivo imaging after using cRGD-modified QD encoded barcodes. (b) Multiplex in vivo imaging of a living mouse by using the SERS barcodes. (c) The process of indexing large amounts of cells and sequencing their RNAs with the droplet microfluidic device. (d) Molecular imaging of single cancer cells by using different aptamer-modified QDs. (a) Reprinted with permission from Ref. [233]. Copyright 2012, the Royal Society of Chemistry. (b) Reprinted with permission from Ref. [235]. Copyright 2009, National Academy of Sciences. (c) Reprinted with permission from Ref. [238]. Copyright 2015, Elsevier Inc. (d) Reprinted with permission from Ref. [240]. Copyright 2009, Wiley$\mathrm{VCH}$.

separated the two streams into discontinuous droplets. One of the streams contained cells, and the other one contained primer-modified barcodes suspended in a lysis buffer. After the formation of the droplets, the cells would be lysed by the lysis buffer and then released their microRNAs, resulting in the specific hybridization with the primers of the barcodes. Next, the stabilization of the droplet surfaces was destroyed by using a reagent and the large number of microRNAs and the barcodes in the droplets were released. Following the reverse transcription of the microRNAs, the PCR handle was introduced downstream of the synthesized cDNAs. Besides the analyses of cell types and cell growths, this technology also can be employed to give the deeper understanding about the specific roles of different kinds of genes in different types of cells.

The multiplexed imaging that can target three different molecular markers simultaneously in a single cancer cell is also helpful for diagnosing cancers at very early stages and learning more knowledges about the oncogenesis of cancers. For example, three different QDs were used to evaluate different proteins in cancer cells. These QDs were modified with aptamers, AS1411, TTA1 and MUC1 , and they can bind to specific molecules of cancer cells [240]. As demonstrated in Fig. 14d, triple cellular imaging of a single cell could be performed by confocal microscopy imaging, the fluorescent signals showed a wide overlap of QD-AS1411 and QD-MUC-1 in all cancer cells, and of all these three QD-conjugated aptamers in C6 and HeLa cells, indicating that co-expression and colocalization of these three different proteins might be very essential for the oncogenesis.

\section{Cell capture}

With the ability to evaluate the function of different cells and detect the abnormal cells in the body fluid, cell 
capture has become a promising method for immune cell analysis, dysfunctional blood cell detection and cancer diagnosis. There have been several strategies developed for capturing cells, such as micro-structures, trapping arrays and microfiltering microfluidic technologies $[241,242]$. However, these strategies usually capture simple cells instead of multiple cell types, which is essential for disease diagnosis. Therefore, it is highly desirable to develop a technique to allow capturing and distinguishing multiplex cells simultaneously. To realize this purpose, a new type of modified polyacrylamide (PAAm) hydrogel PhC barcode particles has been developed to realize simultaneous capture of several kinds of blood cells (Fig. 15a) [243]. Because of the hydrophilia of PAAm hydrogel, such hydrogel barcodes could be modified with different kinds of plasma proteins, such as fibronectin (FN) and phytohemagglutinin (PHA), and as FN could connect with glycoprotein on the platelet membrane and PHA can agglutinate all human erythrocytes, the PAAm barcode particles modified with FN or PHA could capture specific blood cells from the whole blood sample. In this study, red and blue barcodes with their unique reflection peak positions at 660 and $490 \mathrm{~nm}$ were modified with FN and PHA, respectively, whereas the orange ones without any modification were used to be the control group, and the results showed that these modified barcode particles could indeed realize simultaneous capture of several kinds of blood cells from a complex sample, respectively. In addition to $\mathrm{FN}$ and PHA, antibodies, such as anti-EGFR and anti-HER2 which can bind specific kinds of cancer cells, are also used for multiplex cell capture by conjugating them to different SERS barcodes with cyanine and triphenylmethine Raman reporters [244].

Circulating tumor cells (CTC), as another tumor biomarkers, have been extracted from blood samples of patients with many solid cancers. It has proposed that CTC can serve as an early indicator for metastatic cancers and these cells can reflect molecular features of cells within tumor masses. Thus, the detection of CTC considered a real-time "liquid biopsy" for patients with cancer has become a recent emphasis in cancer prognosis and diagnosis [245-249]. With the development of cell capture, CTC can be detected via different technologies that take advantage of their physical and biological properties, including protein-based and mRNA-based technologies. A study has been presented for multiplex circulating tumor cells capture based on the aptamer-modified colloidal crystal barcode particles to help diagnosis of lymphoma and leukemia (Fig. 15b) [250]. Three kinds of barcodes with the unique reflection peak positions at 661,583 , and $447 \mathrm{~nm}$ were coated with three different kinds of aptamers, called TD05, Sgc8, and Sgd5. After the different circulating tumor cells have been captured in real clinical samples, the cells were treated with fluorescently labeled specific antibodies, and then they were analyzed with the flow cytometer after being released from the barcodes. The results showed that about $92.83 \%$ of circulating tumor cells were captured by the barcodes, resulting in the decrease of the amount of cells in the samples from 5.3\% to $0.38 \%$. In addition, due to the specificity between the CTCs and their aptamers on the barcodes, $96.8 \%$ of the cells captured by the red barcodes were CCRF-CEM cells and $98.94 \%$ of the cells captured by the green barcodes were Ramos cells. This approach may become a promising technology for clinical diagnosis and prognosis of cancers.

\section{Cell culture and drug research}

Because of the presence of biomaterials, those with good biocompatibility are used to cultivate cells by being immobilized on the substrate, and the cell culture capacity of the biomaterial can be assessed directly according to the growth states of the cells cultured on it, thus further accelerating the development of cell culture and drug research. In addition, because the biomaterials are mostly plane and static, microcarriers with three-dimensional structures have been used to serve as biomimic platforms for cell culture and better evaluation of these biomaterials, among which encoded barcode particles that can be used to realize the multiplex evaluation of biomaterials have been attracting increasing interest in these fields. Unlike some barcode particles whose encoded information will become unreadable when the target cells are cultured on their surfaces, $\mathrm{PhC}$ barcodes have very stable codes even when their surfaces are covered with the cultured cells. As demonstrated in Fig. 15c, three kinds of silica hybrid $\mathrm{PhC}$ barcode particles with red, green or blue colors were employed for multiple evaluation of biomaterials by HepG2 cells culture [251]. In this study, the red ones were constructed by employing PEG hydrogel and the green ones were constructed with both calcium alginate hydrogel and collagen, while the blue ones without any biomaterials were used as the control group. After adding them into the same microplate, all of them were used for HepG2 cells culture. The results showed that most of HepG2 cells were observed on the green barcodes, whereas only a few cells were on the blue barcodes and even no cells observed on the red barcodes. These properties made the encoded barcodes good platforms for 

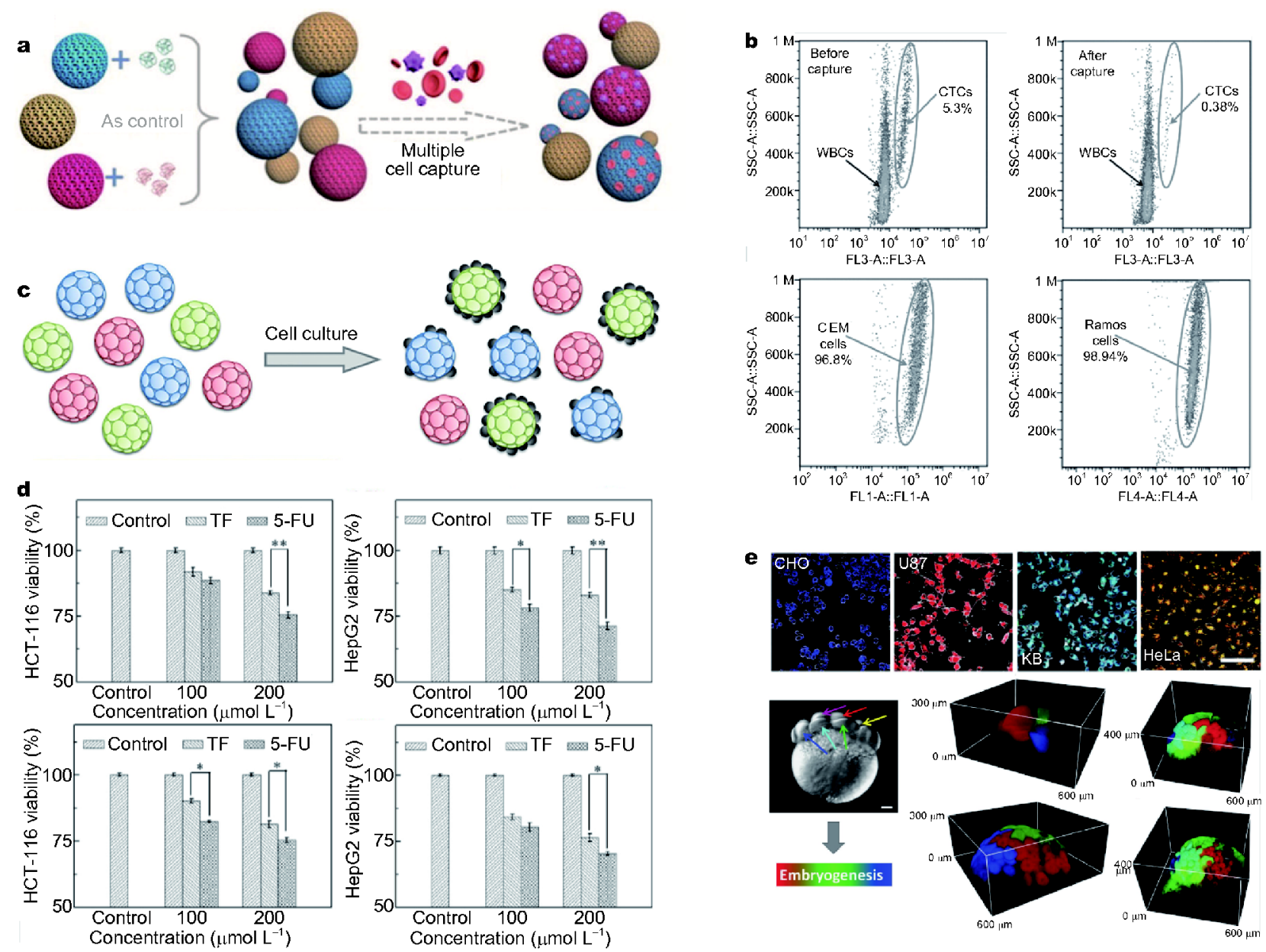

Figure 15 (a) Schematic of multiple blood cells capture with the PhC barcodes. (b) The flow cytometry of the CTCs and white blood cells in the sample before and after cell capture, and the flow cytometry of the cells released from the barcodes. (c) Schematic of multiplex assessment of different biomaterials by HepG2 cells culture on the PhC barcode particles. (d) The results of TF and 5-FU drug research by HepG2 cells culture on the GelMAencapsulated PhC barcode particles. (e) Confocal images of different cell lines labeled with different fluorescent polymer nanoparticles, respectively, and the imaging during the development of zebrafish embryos labeled with fluorescent polymer nanoparticles. (a) Reprinted with permission from Ref. [243]. Copyright 2016, the Royal Society of Chemistry. (b, c) Reprinted with permission from Ref. [250,251]. Copyright 2014 and 2013 , WileyVCH. (d) Reprinted with permission from Ref. [252]. Copyright 2016, American Chemical Society. (e) Reprinted with permission from Ref. [253]. Copyright 2017, Wiley-VCH.

multiplex evaluation of biomaterials, cell culture and drug research.

In addition to the multiple evaluation of biomaterials, novel core-shell methacrylated gelatin (GelMA) hydrogel $\mathrm{PhC}$ barcodes were employed to complete multiplex cells culture and drug research [252]. Unlike the traditional cell culture experiments that cannot meet the demands for mimicing the in-vivo metabolic process of drugs and clarifying the comprehensive impacts of drugs on different tissues, the hydrogel shell of the barcodes could mimic the extracellular environment to help cell culture and the $\mathrm{PhC}$ core of the barcodes could provide stable coding with the unique reflection peak to distinguish the type of cells cultured on the barcodes and their different responses to drug test. As presented in Fig. 15d, the cell viability was significantly decreased by tegafur $(\mathrm{TF})$ when both HCT-116 and HepG2 cells were cultured on the barcodes, compared with that in a TF solution when only HCT-116 cells were cultured on the barcode particles. This result concluded that HepG2 cell spheroids loaded on the barcodes could mimic some liver functions effectively, for example, the spheroids could synthesize P450 enzyme to transform noncytotoxic TF into cytotoxic 5fluorouracil (5-FU), with the prospect of this method in mimicing biosystems effectively and discovering complex metabolic porcesses of drugs accurately. 


\section{Cell tracking}

Long-term fluorescence staining of living cells in multiple colors is of high importance for tracking different cell populations in vitro and in vivo, and in this way helps to address key questions in cancer research, cell differentiation, cell therapy, regenerative medicine, embryogenesis and drug research. For these ideas, by using different color codes, fluorescent polymer nanoparticles have been developed to label and track living cells for the long term (Fig. 15e) [253]. With the advantages of uniform $40 \mathrm{~nm}$ size and surface characteristics, these nanoparticles were endocytosed equally well by 7 cell lines (HeLa, KB, embryonic kidney (293T), Chinese hamster ovary, rat basophilic leucemia, U97, and D2A1) and these color codes could be transmitted to daughter cells in equal proportions, which was helpful for further cell research, such as tracing the distribution of cells throughout the whole zebrafish embryo. Moreover, this method also has been used to distinguish the behavior of HeLa cells that underwent different doxorubicin treatments in direct competition for adhesion to the surface, which was helpful for other drug research.

\section{SUMMARY AND OUTLOOK}

Here, we have provided a comprehensive review of the current development of suspension arrays based on various barcode particles and their applications for multiplex bioassays in disease diagnosis, food and environmental safety, cell tracking, and drug research. However, there are still some problems and limitations of barcode particles-based suspension array technologies, which subsequently limit their practical applications. For example, fluorescent encoding barcodes have the disadvantages of photobleaching, limited encoding ability and multi-excitation light uses; UCNPs are usually filled with hydrophobic ligands which limit their biological applications; SERS encoding barcodes also have limited encoding ability; shape encoding barcodes need complex decoding procedures and equipment. Thanks to the rapid development of technology, tremendous progress has been made to solve these problems in recent years, for example, the encoding number of fluorescent barcodes can be increased by mixing different organic dyes with various ratios; novel QDs with NIR emission can avoid photobleaching; fluorescent barcodes with multiple emissions under one single excitation can be obtained by fluorescence resonance energy transfer; hydrophilic surface can be generated by incorporating UCNPs with polymer particles to realize bio-applications of UCNPs; SERS barcodes with different wavenumber and intensity ratio can be obtained by using different reporters and changing their molecular ratio; and by using dual mode encoding technology, the encoding number also can be greater.

Moreover, among the various barcode particles, only optical-especially fluorescent-barcode particles have been used widely in multiplex bioassays, and most of them remain in the laboratory, facing challenges to meet the requirements of real applications. To overcome such challenges, some issues should be considered in developing applications. First, to achieve mass production and commercialization of particles, several improvements are needed, such as shortening the time-consuming process of synthesis, increasing the uniformity of synthesized barcodes, and automation to streamline the synthesis process. Second, in view of the multiplicity of targets, much emphasis should be put not only on the encoding number of particles, which is a considerable problem for the application of nonoptical particles, but also on the need for multifunctional barcodes for convenient assays. For example, those with magnetic properties are being explored extensively for easy separation and mixing. Besides improving functionality, the third issue concerns the structure, which could reduce nonspecific adsorption for lower false-positive readings while enhancing the reaction kinetics for higher sensitivity. In addition to barcode particles, the strategies of reaction methods, such as the sandwich immunoassays that are the most widely used, also need to be optimized for reducing nonspecific reactions between molecules. Moreover, miniaturization of automatic systems that can produce high-throughput assays as well as the decoding of multiplex information are further requirements to be met in achieving point-ofcare applications for mass market implementation.

Suspension arrays based on appropriate barcode particles are mainly used in clinical applications for disease diagnosis, such as for cancers, heart diseases, infectious diseases, and Down syndrome; however, there are many other diseases that have not yet been studied. At present, large numbers of studies based on multiple proteins, genes, and cells have been performed for cancer screening, while other diseases are only screened at the single protein or gene level. Thus, attempts should be made to widen multiplex detection by using more biomarkers for each disease and thereby provide more accurate diagnosis. Moreover, the clinical application of barcode particles could enter a new chapter by realizing multiplex detection at three levels in one reaction system. With the development of personalized medication, research on novel drugs is also important and warrants more interest 
from researchers. Finally, it is important for the barcode particles to be appropriately used in more research fields, such as in the clinical, medicinal, nutritional, and environmental fields, and implementation of the technology for mass markets needs to be regulated by the authorities to ensure the safe usage of these barcodes. With further efforts from researchers in addressing these challenges, the huge potential of barcode particles can certainly be realized for a wide variety of applications.

\section{Received 1 June 2018; accepted 29 July 2018;}

published online 10 September 2018

1 McLaughlin JL, Rogers LL, Anderson JE. The use of biological assays to evaluate botanicals. Drug Inf J, 1998, 32: 513-524

2 Robinson J. Bioassays-a continuously developing field. Drug Discovery Today, 2003, 8: 676-678

3 Nolan JP, Sklar LA. Suspension array technology: evolution of the flat-array paradigm. Trends Biotech, 2002, 20: 9-12

4 Leng Y, Sun K, Chen X, et al. Suspension arrays based on nanoparticle-encoded microspheres for high-throughput multiplexed detection. Chem Soc Rev, 2015, 44: 5552-5595

5 Chin L, Andersen JN, Futreal PA. Cancer genomics: from discovery science to personalized medicine. Nat Med, 2011, 17: 297303

6 Lander ES. Initial impact of the sequencing of the human genome. Nature, 2011, 470: 187-197

7 Braeckmans K, De Smedt SC, Leblans M, et al. Encoding microcarriers: present and future technologies. Nat Rev Drug Discov, 2002, 1: 447-456

8 Templin MF, Stoll D, Schrenk M, et al. Protein microarray technology. Drug Discovery Today, 2002, 7: 815-822

9 Wilson R, Cossins AR, Spiller DG. Encoded microcarriers for high-throughput multiplexed detection. Angew Chem Int Ed, 2006, 45: 6104-6117

10 Rao RS, Visuri SR, McBride MT, et al. Comparison of multiplexed techniques for detection of bacterial and viral proteins. J Proteome Res, 2004, 3: 736-742

11 Birtwell S, Morgan H. Microparticle encoding technologies for high-throughput multiplexed suspension assays. Integr Biol, 2009, 1: 345

12 Houser B. Bio-Rad's Bio-Plex suspension array system, xMAP technology overview. Archives Physiol Biochem, 2012, 118: 192196

13 Dunbar SA. Applications of Luminex $\mathrm{xMAP}^{\mathrm{TM}}$ technology for rapid, high-throughput multiplexed nucleic acid detection. Clinica Chim Acta, 2006, 363: 71-82

14 Xie Z, Cao K, Zhao Y, et al. An optical nose chip based on mesoporous colloidal photonic crystal beads. Adv Mater, 2014, 26: 2413-2418

15 Shikha S, Salafi T, Cheng J, et al. Versatile design and synthesis of nano-barcodes. Chem Soc Rev, 2017, 46: 7054-7093

16 Zhao Y, Zhao X, Gu Z. Photonic crystals in bioassays. Adv Funct Mater, 2010, 20: 2970-2988

17 Porter MD, Lipert RJ, Siperko LM, et al. SERS as a bioassay platform: fundamentals, design, and applications. Chem Soc Rev, 2008, 37: 1001-1011

18 Zhao Y, Shang L, Cheng Y, et al. Spherical colloidal photonic crystals. Acc Chem Res, 2014, 47: 3632-3642

19 Wang H, Gu H, Chen Z, et al. Enzymatic inverse opal hydrogel particles for biocatalyst. ACS Appl Mater Interfaces, 2017, 9: 12914-12918

20 Carter JM, Lin A, Clotilde L, et al. Rapid, multiplexed characterization of shiga toxin-producing Escherichia coli (STEC) isolates using suspension array technology. Front Microbiol, 2016, 7: 439

21 Sun Z, Peng Y, Zhang M, et al. Simultaneous and highly sensitive detection of six different foodborne pathogens by high-throughput suspension array technology. Food Control, 2014, 40: 300309

22 Wang H, Shang LR, Gu XX, et al. The preparation and biomedical applications of encoded microcarriers. Prog Chem, 2017, 29: 1159-1172

23 Toro M, Najjar MB, Ju W, et al. Molecular serogrouping of Shiga toxin-producing escherichia coli using suspension array. Foodborne Pathogens Dis, 2013, 10: 478-480

24 Zhao Y, Cheng Y, Shang L, et al. Microfluidic synthesis of barcode particles for multiplex assays. Small, 2015, 11: 151-174

25 Behnke T, Würth C, Hoffmann K, et al. Encapsulation of hydrophobic dyes in polystyrene micro- and nanoparticles via swelling procedures. J Fluoresc, 2011, 21: 937-944

26 Wang L, Yang C, Tan W. Dual-luminophore-doped silica nanoparticles for multiplexed signaling. Nano Lett, 2005, 5: 37-43

27 Li Y, Cu YTH, Luo D. Multiplexed detection of pathogen DNA with DNA-based fluorescence nanobarcodes. Nat Biotechnol, 2005, 23: 885-889

28 Wang L, Tan W. Multicolor FRET silica nanoparticles by single wavelength excitation. Nano Lett, 2006, 6: 84-88

29 Mei J, Leung NLC, Kwok RTK, et al. Aggregation-induced emission: together we shine, united we soar! Chem Rev, 2015, 115: $11718-11940$

30 Han M, Gao X, Su JZ, et al. Quantum-dot-tagged microbeads for multiplexed optical coding of biomolecules. Nat Biotechnol, 2001, 19: 631-635

31 Wang HQ, Wang JH, Li YQ, et al. Multi-color encoding of polystyrene microbeads with $\mathrm{CdSe} / \mathrm{ZnS}$ quantum dots and its application in immunoassay. J Colloid Interface Sci, 2007, 316: 622-627

32 Riegler J, Ehlert O, Nann T. A facile method for coding and labeling assays on polystyrene beads with differently colored luminescent nanocrystals. Anal Bioanal Chem, 2006, 384: 645-650

33 Wang X, Ma Q, Li B, et al. The preparation of CdTe nanoparticles and CdTe nanoparticle-labelled microspheres for biological applications. Luminescence, 2007, 22: 1-8

34 Rauf S, Glidle A, Cooper JM. Production of quantum dot barcodes using biological self-assembly. Adv Mater, 2009, 21: 40204024

35 Song F, Tang PS, Durst H, et al. Nonblinking plasmonic quantum dot assemblies for multiplex biological detection. Angew Chem Int Ed, 2012, 51: 8773-8777

36 Wilson R, Spiller DG, Prior IA, et al. A simple method for preparing spectrally encoded magnetic beads for multiplexed detection. ACS Nano, 2007, 1: 487-493

37 Wang Q, Seo DK. Preparation of photostable quantum dotpolystyrene microbeads through covalent organosilane coupling of CdSe@ZnS quantum dots. J Mater Sci, 2009, 44: 816-820

38 Vaidya SV, Gilchrist ML, Maldarelli C, et al. Spectral bar coding of polystyrene microbeads using multicolored quantum dots. 
Anal Chem, 2007, 79: 8520-8530

39 Song T, Liu J, Li W, et al. Self-healing encapsulation strategy for preparing highly stable, functionalized quantum-dot barcodes. ACS Appl Mater Interfaces, 2014, 6: 2745-2752

40 Wang G, Leng Y, Dou H, et al. Highly efficient preparation of multiscaled quantum dot barcodes for multiplexed hepatitis $\mathrm{B}$ detection. ACS Nano, 2013, 7: 471-481

41 Liu H, Qian X, Wu Z, et al. Microfluidic synthesis of QD-encoded PEGDA microspheres for suspension assay. J Mater Chem B, 2016, 4: 482-488

42 Yeom SY, Son CH, Kim BS, et al. Multiplexed detection of epigenetic markers using quantum dot (QD)-encoded hydrogel microparticles. Anal Chem, 2016, 88: 4259-4268

43 Wang X, Wang G, Li W, et al. NIR-emitting quantum dot-encoded microbeads through membrane emulsification for multiplexed immunoassays. Small, 2013, 30

44 Zhang Y, Dong C, Su L, et al. Multifunctional microspheres encoded with upconverting nanocrystals and magnetic nanoparticles for rapid separation and immunoassays. ACS Appl Mater Interfaces, 2016, 8: 745-753

45 Chen C, Zhang P, Gao G, et al. Near-infrared-emitting two-dimensional codes based on lattice-strained core/(doped) shell quantum dots with long fluorescence lifetime. Adv Mater, 2014, 26: 6313-6317

46 Wang H, Liu Z, Wang S, et al. MC540 and upconverting nanocrystal coloaded polymeric liposome for near-infrared light-triggered photodynamic therapy and cell fluorescent imaging. ACS Appl Mater Interfaces, 2014, 6: 3219-3225

47 Jiang S, Zhang Y, Lim KM, et al. NIR-to-visible upconversion nanoparticles for fluorescent labeling and targeted delivery of siRNA. Nanotechnology, 2009, 20: 155101

48 Huang K, Idris NM, Zhang Y. Engineering of lanthanide-doped upconversion nanoparticles for optical encoding. Small, 2016, 12: 836-852

49 Auzel F. Upconversion and anti-Stokes processes with $\mathrm{f}$ and $\mathrm{d}$ ions in solids. Chem Rev, 2004, 104: 139-174

50 Page RH, Schaffers KI, Waide PA, et al. Upconversion-pumped luminescence efficiency of rare-earth-doped hosts sensitized with trivalent ytterbium. J Opt Soc Am B, 1998, 15: 996-1008

51 You M, Lin M, Wang S, et al. Three-dimensional quick response code based on inkjet printing of upconversion fluorescent nanoparticles for drug anti-counterfeiting. Nanoscale, 2016, 8: 10096-10104

52 Zhang Y, Zhang L, Deng R, et al. Multicolor barcoding in a single upconversion crystal. J Am Chem Soc, 2014, 136: 4893-4896

53 You M, Zhong J, Hong Y, et al. Inkjet printing of upconversion nanoparticles for anti-counterfeit applications. Nanoscale, 2015, 7: 4423-4431

54 Rantanen $\mathrm{T}$, Järvenpää $\mathrm{ML}$, Vuojola $\mathrm{J}$, et al. Fluorescencequenching-based enzyme-activity assay by using photon upconversion. Angew Chem Int Ed, 2008, 47: 3811-3813

55 Kim WJ, Nyk M, Prasad PN. Color-coded multilayer photopatterned microstructures using lanthanide (III) ion co-doped $\mathrm{NaYF}_{4}$ nanoparticles with upconversion luminescence for possible applications in security. Nanotechnology, 2009, 20: 185301

56 Zhang F, Haushalter RC, Haushalter RW, et al. Rare-earth upconverting nanobarcodes for multiplexed biological detection. Small, 2011, 7: 1972-1976

57 Zhang F, Shi Q, Zhang Y, et al. Fluorescence upconversion microbarcodes for multiplexed biological detection: nucleic acid encoding. Adv Mater, 2011, 45

58 Gorris HH, Ali R, Saleh SM, et al. Tuning the dual emission of photon-upconverting nanoparticles for ratiometric multiplexed encoding. Adv Mater, 2011, 23: 1652-1655

59 Zhang X, Ren Y, Chen M, et al. Fabrication of polystyrene/upconversion nanocrystals nanocomposite spheres through in situ dispersion polymerization. J Colloid Interface Sci, 2011, 358: 347353

60 Chen $\mathrm{Z}$, Chen $\mathrm{H}, \mathrm{Hu} \mathrm{H}$, et al. Versatile synthesis strategy for carboxylic acid-functionalized upconverting nanophosphors as biological labels. J Am Chem Soc, 2008, 130: 3023-3029

61 Boyer JC, Manseau MP, Murray JI, et al. Surface modification of upconverting $\mathrm{NaYF}_{4}$ nanoparticles with PEG-phosphate ligands for NIR (800 $\mathrm{nm})$ biolabeling within the biological window. Langmuir, 2010, 26: 1157-1164

62 An M, Cui J, He Q, et al. Down-/up-conversion luminescence nanocomposites for dual-modal cell imaging. J Mater Chem B, 2013, 1: 1333-1339

63 Zhang H, Li Y, Ivanov IA, et al. Plasmonic modulation of the upconversion fluorescence in $\mathrm{NaYF}_{4}: \mathrm{Yb} / \mathrm{Tm}$ hexaplate nanocrystals using gold nanoparticles or nanoshells. Angew Chem Int Ed, 2010, 49: 2865-2868

64 Liu Q, Chen M, Sun Y, et al. Multifunctional rare-earth selfassembled nanosystem for tri-modal upconversion luminescence/ fluorescence/positron emission tomography imaging. Biomaterials, 2011, 32: 8243-8253

65 Li Z, Zhang Y. Monodisperse silica-coated polyvinylpyrrolidone/ $\mathrm{NaYF}_{4}$ nanocrystals with multicolor upconversion fluorescence emission. Angew Chem Int Ed, 2006, 45: 7732-7735

66 Gnach A, Bednarkiewicz A. Lanthanide-doped up-converting nanoparticles: Merits and challenges. Nano Today, 2012, 7: 532563

67 Generalova AN, Kochneva IK, Khaydukov EV, et al. Submicron polyacrolein particles in situ embedded with upconversion nanoparticles for bioassay. Nanoscale, 2015, 7: 1709-1717

68 Shang L, Gu Z, Zhao Y. Structural color materials in evolution. Mater Today, 2016, 19: 420-421

69 Lee SS, Kim SK, Won JC, et al. Reconfigurable photonic capsules containing cholesteric liquid crystals with planar alignment. Angew Chem Int Ed, 2015, 54: 15266-15270

70 Zhao Y, Xie Z, Gu H, et al. Bio-inspired variable structural color materials. Chem Soc Rev, 2012, 41: 3297-3317

71 Sim JY, Lee GH, Kim SH. Microfluidic design of magnetoresponsive photonic microcylinders with multicompartments. Small, 2015, 11: 4938-4945

72 Darragh PJ, Gaskin AJ, Terrell BC, et al. Origin of precious opal. Nature, 1966, 209: 13-16

73 Srinivasarao M. Nano-optics in the biological world: beetles, butterflies, birds, and moths. Chem Rev, 1999, 99: 1935-1962

74 Phillips KR, England GT, Sunny S, et al. A colloidoscope of colloid-based porous materials and their uses. Chem Soc Rev, 2016, 45: 281-322

75 Zhao Y, Zhao X, Sun C, et al. Encoded silica colloidal crystal beads as supports for potential multiplex immunoassay. Anal Chem, 2008, 80: 1598-1605

76 Kanai T, Lee D, Shum HC, et al. Fabrication of tunable spherical colloidal crystals immobilized in soft hydrogels. Small, 2010, 6: 807-810

77 Fenzl C, Hirsch T, Wolfbeis OS. Photonic crystals for chemical sensing and biosensing. Angew Chem Int Ed, 2014, 53: 3318- 
3335

78 Ge J, Yin Y. Responsive photonic crystals. Angew Chem Int Ed, 2011, 50: 1492-1522

79 Cai Z, Smith NL, Zhang JT, et al. Two-dimensional photonic crystal chemical and biomolecular sensors. Anal Chem, 2015, 87: 5013-5025

80 Kanai T, Lee D, Shum HC, et al. Gel-immobilized colloidal crystal shell with enhanced thermal sensitivity at photonic wavelengths. Adv Mater, 2010, 22: 4998-5002

81 Ge J, Yin Y. Magnetically responsive colloidal photonic crystals. J Mater Chem, 2008, 18: 5041-5045

82 Mao Z, Xu H, Wang D. Molecular mimetic self-assembly of colloidal particles. Adv Funct Mater, 2010, 20: 1053-1074

83 Zhao Y, Zhao X, Tang B, et al. Rapid and sensitive biomolecular screening with encoded macroporous hydrogel photonic beads. Langmuir, 2010, 26: 6111-6114

84 Bai S, Nguyen TL, Mulvaney $\mathrm{P}$, et al. Using hydrogels to accommodate hydrophobic nanoparticles in aqueous media via solvent exchange. Adv Mater, 2010, 22: 3247-3250

85 Chen H, Lou R, Chen Y, et al. Photonic crystal materials and their application in biomedicine. Drug Deliver, 2017, 24: 775-780

86 Lifson MA, Miller BL. Photonic crystals as robust label-free biosensors. Springer Ser Mater Sci, 2015, 229: 189-207

87 Li Y, Zhou X, Yang Q, et al. Patterned photonic crystals for hiding information. J Mater Chem C, 2017, 5: 4621-4628

88 Josephson DP, Miller $\mathrm{M}$, Stein A. Inverse opal $\mathrm{SiO}_{2}$ photonic crystals as structurally-colored pigments with additive primary colors. Z Anorg Allg Chem, 2014, 640: 655-662

89 Shang L, Shangguan F, Cheng Y, et al. Microfluidic generation of magnetoresponsive Janus photonic crystal particles. Nanoscale, 2013, 5: 9553-9557

90 Shiu JY, Kuo CW, Chen P, et al. Fabrication of tunable superhydrophobic surfaces by nanosphere lithography. Chem Mater, 2004, 16: 561-564

91 Zhao Z, Wang H, Shang L, et al. Bioinspired heterogeneous structural color stripes from capillaries. Adv Mater, 2017, 29: 1704569

92 Shi X, Li M, Ye C, et al. Photonic crystal boosted chemiluminescence reaction. Laser Photonics Rev, 2013, 7: L39-L43

93 Schäfer CG, Smolin DA, Hellmann GP, et al. Fully reversible shape transition of soft spheres in elastomeric polymer opal films. Langmuir, 2013, 29: 11275-11283

94 Gu H, Rong F, Tang B, et al. Photonic crystal beads from gravitydriven microfluidics. Langmuir, 2013, 29: 7576-7582

95 Zhou M, Bao J, Xu Y, et al. Photoelectrodes based upon Mo: $\mathrm{BiVO}_{4}$ Inverse opals for photoelectrochemical water splitting. ACS Nano, 2014, 8: 7088-7098

96 Kim SH, Shim JW, Yang SM. Microfluidic multicolor encoding of microspheres with nanoscopic surface complexity for multiplex immunoassays. Angew Chem Int Ed, 2011, 50: 1171-1174

97 Zhao Y, Xie Z, Gu H, et al. Multifunctional photonic crystal barcodes from microfluidics. NPG Asia Mater, 2012, 4: e25

98 Pikul JH, Gang Zhang H, Cho J, et al. High-power lithium ion microbatteries from interdigitated three-dimensional bicontinuous nanoporous electrodes. Nat Commun, 2013, 4: 1732

99 Fu F, Chen Z, Zhao Z, et al. Bio-inspired self-healing structural color hydrogel. Proc Natl Acad Sci USA, 2017, 114: 5900-5905

100 Qin M, Huang Y, Li Y, et al. A rainbow structural-color chip for multisaccharide recognition. Angew Chem Int Ed, 2016, 55: 6911-6914
101 Lee HS, Shim TS, Hwang H, et al. Colloidal photonic crystals toward structural color palettes for security materials. Chem Mater, 2013, 25: 2684-2690

102 Santos A, Law CS, Pereira T, et al. Nanoporous hard data: optical encoding of information within nanoporous anodic alumina photonic crystals. Nanoscale, 2016, 8: 8091-8100

103 Cheng Y, Zhao Y, Shangguan F, et al. Convenient generation of quantum dot-incorporated photonic crystal beads for multiplex bioassays. J Biomed Nanotechnol, 2014, 10: 760-766

104 Shang L, Fu F, Cheng Y, et al. Photonic crystal microbubbles as suspension barcodes. J Am Chem Soc, 2015, 137: 15533-15539

105 Zhao Y, Zhao X, Pei X, et al. Multiplex detection of tumor markers with photonic suspension array. Anal Chim Acta, 2009, 633: 103-108

106 Li J, Dong S, Tong J, et al. 3D ordered silver nanoshells silica photonic crystal beads for multiplex encoded SERS bioassay. Chem Commun, 2016, 52: 284-287

107 Yan Z, Tian C, Qu X, et al. DNA-functionalized photonic crystal microspheres for multiplex detection of toxic metal ions. Colloids Surfs B-Biointerfaces, 2017, 154: 142-149

108 Ye B, Ding H, Cheng Y, et al. Photonic crystal microcapsules for label-free multiplex detection. Adv Mater, 2014, 26: 3270-3274

109 Zhang Y, Gao L, Wen L, et al. Highly sensitive, selective and reusable mercury(II) ion sensor based on a ssDNA-functionalized photonic crystal film. Phys Chem Chem Phys, 2013, 15: 1194311949

110 Ye B, Rong F, Gu H, et al. Bioinspired angle-independent photonic crystal colorimetric sensing. Chem Commun, 2013, 49: 5331-5333

111 Laing S, Gracie K, Faulds K. Multiplex in vitro detection using SERS. Chem Soc Rev, 2016, 45: 1901-1918

112 Chao J, Cao W, Su S, et al. Nanostructure-based surface-enhanced Raman scattering biosensors for nucleic acids and proteins. J Mater Chem B, 2016, 4: 1757-1769

113 Liu K, Bai Y, Zhang L, et al. Porous Au-Ag nanospheres with high-density and highly accessible hotspots for SERS analysis. Nano Lett, 2016, 16: 3675-3681

114 Mir-Simon B, Reche-Perez I, Guerrini L, et al. Universal one-pot and scalable synthesis of SERS encoded nanoparticles. Chem Mater, 2015, 27: 950-958

115 Lai Y, Sun S, He T, et al. Raman-encoded microbeads for spectral multiplexing with SERS detection. RSC Adv, 2016, 5: 1376213767

116 Zhu D, Wang Z, Zong S, et al. Wavenumber-intensity joint SERS encoding using silver nanoparticles for tumor cell targeting. RSC Adv, 2014, 4: 60936-60942

117 Li R, Zhang Y, Tan J, et al. Dual-mode encoded magnetic composite microsphere based on fluorescence reporters and Raman probes as covert tag for anticounterfeiting applications. ACS Appl Mater Interfaces, 2016, 8: 9384-9394

118 Wang Z, Zong S, Li W, et al. SERS-fluorescence joint spectral encoding using organic-metal-QD hybrid nanoparticles with a huge encoding capacity for high-throughput biodetection: putting theory into practice. J Am Chem Soc, 2012, 134: 2993-3000

119 Gebrekidan MT, Knipfer C, Stelzle F, et al. A shifted-excitation Raman difference spectroscopy (SERDS) evaluation strategy for the efficient isolation of Raman spectra from extreme fluorescence interference. J Raman Spectrosc, 2016, 47: 198-209

120 Nicewarner-Peña SR, Carado AJ, Shale KE, et al. Barcoded metal nanowires: optical reflectivity and patterned fluorescence. J Phys 
Chem B, 2003, 107: 7360-7367

121 Demirok UK, Burdick J, Wang J. Orthogonal multi-readout identification of alloy nanowire barcodes. J Am Chem Soc, 2009, 131: 22-23

122 Sattayasamitsathit S, Burdick J, Bash R, et al. Alloy nanowires bar codes based on nondestructive X-ray fluorescence readout. Anal Chem, 2007, 79: 7571-7575

123 Evans M, Sewter C, Hill E. An encoded particle array tool for multiplex bioassays. ASSAY Drug Dev Technologies, 2003, 1: 199-207

124 Pregibon DC, Toner M, Doyle PS. Multifunctional encoded particles for high-throughput biomolecule analysis. Science, 2007, 315: 1393-1396

125 Cederquist KB, Dean SL, Keating CD. Encoded anisotropic particles for multiplexed bioanalysis. WIREs Nanomed Nanobiotechnol, 2010, 2: 578-600

126 Lee H, Kim J, Kim H, et al. Colour-barcoded magnetic microparticles for multiplexed bioassays. Nat Mater, 2010, 9: 745-749

127 Svedberg G, Jeong Y, Na H, et al. Towards encoded particles for highly multiplexed colorimetric point of care autoantibody detection. Lab Chip, 2017, 17: 549-556

128 He B, Son SJ, Lee SB. Shape-coded silica nanotubes for biosensing. Langmuir, 2006, 22: 8263-8265

129 Meiring JE, Schmid MJ, Grayson SM, et al. Hydrogel biosensor array platform indexed by shape. Chem Mater, 2004, 16: 55745580

130 Kim LN, Kim M, Jung K, et al. Shape-encoded silica microparticles for multiplexed bioassays. Chem Commun, 2015, 51: 12130-12133

131 Han S, Bae HJ, Kim SD, et al. An encoded viral micropatch for multiplex cell-based assays through localized gene delivery. Lab Chip, 2017, 17: 2435-2442

132 Llandro J, Palfreyman JJ, Ionescu A, et al. Magnetic biosensor technologies for medical applications: a review. Med Biol Eng Comput, 2010, 48: 977-998

133 Gaster RS, Hall DA, Nielsen CH, et al. Matrix-insensitive protein assays push the limits of biosensors in medicine. Nat Med, 2009, 15: 1327-1332

134 Yoon SJ, Kim BG, Tak Jeon I, et al. Compositional dependence of magnetic properties in $\mathrm{CoFe} / \mathrm{Au}$ nanobarcodes. Appl Phys Express, 2012, 5: 103003

135 Hong B, Jeong JR, Llandro J, et al. High throughput biological analysis using multi-bit magnetic digital planar tags. AIP Conf Proc, 2008, 1025: 74

136 Love DM, Vyas KN, Fernández-Pacheco A, et al. A composite element bit design for magnetically encoded microcarriers for future combinatorial chemistry applications. RSC Adv, 2015, 5: 10211-10218

137 Pan J, Feng SS. Targeting and imaging cancer cells by folatedecorated, quantum dots (QDs)- loaded nanoparticles of biodegradable polymers. Biomaterials, 2009, 30: 1176-1183

138 Li J, Wang $\mathrm{H}$, Dong S, et al. Quantum-dot-tagged photonic crystal beads for multiplex detection of tumor markers. Chem Commun, 2014, 50: 14589-14592

139 Xie M, Hu J, Wen CY, et al. Fluorescent-magnetic dual-encoded nanospheres: a promising tool for fast-simultaneous-addressable high-throughput analysis. Nanotechnology, 2012, 23: 035602

140 Zhang DS, Jiang $\mathrm{Y}$, Yang $\mathrm{H}$, et al. Dual-encoded microbeads through a host-guest structure: enormous, flexible, and accurate barcodes for multiplexed assays. Adv Funct Mater, 2016, 26:
6146-6157

141 Lu S, Zhang DS, Wei D, et al. Three-dimensional barcodes with ultrahigh encoding capacities: a flexible, accurate, and reproducible encoding strategy for suspension arrays. Chem Mater, 2017, 29: 10398-10408

142 Qing Z, He X, Wang K, et al. Colorimetric multiplexed analysis of mercury and silver ions by using a unimolecular DNA probe and unmodified gold nanoparticles. Anal Methods, 2012, 4: 33203325

143 Wang C, Li X, Zhang F. Bioapplications and biotechnologies of upconversion nanoparticle-based nanosensors. Analyst, 2016, 141: $3601-3620$

144 Chen J, Li Y, Lv K, et al. Cyclam-functionalized carbon dots sensor for sensitive and selective detection of copper(II) ion and sulfide anion in aqueous media and its imaging in live cells. Sensor Actuat B-Chem, 2016, 224: 298-306

145 Colon M, Todolí JL, Hidalgo M, et al. Development of novel and sensitive methods for the determination of sulfide in aqueous samples by hydrogen sulfide generation-inductively coupled plasma-atomic emission spectroscopy. Anal Chim Acta, 2008, 609: $160-168$

146 Eto K, Asada T, Arima K, et al. Brain hydrogen sulfide is severely decreased in Alzheimer's disease. Biochem BioPhys Res Commun, 2002, 293: 1485-1488

147 Zuo P, Yin BC, Ye BC. DNAzyme-based microarray for highly sensitive determination of metal ions. Biosens Bioelectron, 2009, 25: 935-939

148 Tahán JE, Granadillo VA, Romero RA. Electrothermal atomic absorption spectrometric determination of $\mathrm{Al}, \mathrm{Cu}, \mathrm{Fe}, \mathrm{Pb}, \mathrm{V}$ and $\mathrm{Zn}$ in clinical samples and in certified environmental reference materials. Anal Chim Acta, 1994, 295: 187-197

149 Etienne M, Bessiere J, Walcarius A. Voltammetric detection of copper(II) at a carbon paste electrode containing an organically modified silica. Senss Actuators B-Chem, 2001, 76: 531-538

150 Liu HW, Jiang SJ, Liu SH. Determination of cadmium, mercury and lead in seawater by electrothermal vaporization isotope dilution inductively coupled plasma mass spectrometry. SpectroChim Acta Part B-Atomic Spectr, 1999, 54: 1367-1375

151 Liu F, Ha HD, Han DJ, et al. Photoluminescent graphene oxide microarray for multiplex heavy metal ion analysis. Small, 2013, 9: 3410-3414

152 Zhang Y, Zuo P, Ye BC. A low-cost and simple paper-based microfluidic device for simultaneous multiplex determination of different types of chemical contaminants in food. Biosens Bioelectron, 2015, 68: 14-19

153 Yan H, Tang NM, Jairo GA, et al. High-sensitivity highthroughput chip based biosensor array for multiplexed detection of heavy metals. Proc SPIE, 2016, 9725

154 Gao W, Nyein HYY, Shahpar Z, et al. Wearable microsensor array for multiplexed heavy metal monitoring of body fluids. ACS Sens, 2016, 1: 866-874

155 Zhou Y, Tang L, Zeng G, et al. Current progress in biosensors for heavy metal ions based on DNAzymes/DNA molecules functionalized nanostructures: A review. Sensor Actuat B-Chem, 2016, 223: $280-294$

156 Liu M, Zhao H, Chen S, et al. A "turn-on" fluorescent copper biosensor based on DNA cleavage-dependent graphene-quenched DNAzyme. Biosens Bioelectron, 2011, 26: 4111-4116

157 He Y, Tian J, Zhang J, et al. DNAzyme self-assembled gold nanorods-based FRET or polarization assay for ultrasensitive and 
selective detection of copper(II) ion. Biosens Bioelectron, 2014, 55: $285-288$

158 Clever GH, Kaul C, Carell T. DNA-metal base pairs. Angew Chem Int Ed, 2007, 46: 6226-6236

159 Willner I, Zayats M. Electronic aptamer-based sensors. Angew Chem Int Ed, 2007, 46: 6408-6418

160 Liu J, Cao Z, Lu Y. Functional nucleic acid sensors. Chem Rev, 2009, 109: 1948-1998

161 Wang Z, Heon Lee J, Lu Y. Highly sensitive "turn-on" fluorescent sensor for $\mathrm{Hg}^{2+}$ in aqueous solution based on structure-switching DNA. Chem Commun, 2008, 172: 6005-6007

162 Wu CS, Khaing Oo MK, Fan X. Highly sensitive multiplexed heavy metal detection using quantum-dot-labeled DNAzymes. ACS Nano, 2010, 4: 5897-5904

163 Li D, Wieckowska A, Willner I. Optical Analysis of $\mathrm{Hg}^{2+}$ ions by oligonucleotide-gold-nanoparticle hybrids and DNA-based machines. Angew Chem Int Ed, 2008, 47: 3927-3931

164 Freeman R, Finder T, Willner I. Multiplexed analysis of $\mathrm{Hg}^{2+}$ and $\mathrm{Ag}^{+}$ions by nucleic acid functionalized CdSe/ZnS quantum dots and their use for logic gate operations. Angew Chem Int Ed, 2009, 48: 7818-7821

165 del Mercato LL, Abbasi AZ, Ochs M, et al. Multiplexed sensing of ions with barcoded polyelectrolyte capsules. ACS Nano, 2011, 5: 9668-9674

166 Ashraf S, Carrillo-Carrion C, Zhang Q, et al. Fluorescence-based ion-sensing with colloidal particles. Curr Opin Pharmacol, 2014, 18: $98-103$

167 Li S, Xu L, Ma W, et al. Triple Raman label-encoded gold nanoparticle trimers for simultaneous heavy metal ion detection. Small, 2015, 11: 3435-3439

168 Zeng Y, Ren J, Shen A, et al. Field and pretreatment-free detection of heavy-metal ions in organic polluted water through an alkyne-coded SERS test kit. ACS Appl Mater Interfaces, 2016, 8: 27772-27778

169 Yu L, Liu X, Yuan W, et al. Confined flocculation of ionic pollutants by poly(l-dopa)-based polyelectrolyte complexes in hydrogel beads for three-dimensional, quantitative, efficient water decontamination. Langmuir, 2015, 31: 6351-6366

170 Culha M. Surface-enhanced Raman scattering: An emerging label-free detection and identification technique for proteins. Appl Spectrosc, 2013, 67: 355-364

171 Zhang H, Zhao Q, Li XF, et al. Ultrasensitive assays for proteins. Analyst, 2007, 132: 724-737

172 Jun BH, Kang H, Lee YS, et al. Fluorescence-based multiplex protein detection using optically encoded microbeads. Molecules, 2012, 17: 2474-2490

173 Gstaiger M, Aebersold R. Applying mass spectrometry-based proteomics to genetics, genomics and network biology. Nat Rev Genet, 2009, 10: 617-627

174 Han X, Aslanian A, Yates Iii JR. Mass spectrometry for proteomics. Curr Opin Chem Biol, 2008, 12: 483-490

175 Armstrong EG, Ehrlich PH, Birken S, et al. Use of a highly sensitive and specific immunoradiometric assay for detection of human chorionic gonadotropin in urine of normal, nonpregnaiit, and pregnant individuals. J Clinical Endocrinology Metabolism, 1984, 59: 867-874

176 Grossman HB, Messing E, Soloway M, et al. Detection of bladder cancer using a point-of-care proteomic assay. JAMA, 2005, 293: 810-816

177 Herbáth M, Papp K, Balogh A, et al. Exploiting fluorescence for multiplex immunoassays on protein microarrays. Methods Appl Fluoresc, 2014, 2: 032001

178 Kingsmore SF. Multiplexed protein measurement: technologies and applications of protein and antibody arrays. Nat Rev Drug Discov, 2006, 5: 310-321

179 Rousserie G, Sukhanova A, Even-Desrumeaux K, et al. Semiconductor quantum dots for multiplexed bio-detection on solidstate microarrays. Critical Rev Oncology/Hematology, 2010, 74: $1-15$

180 Bock C, Coleman M, Collins B, et al. Photoaptamer arrays applied to multiplexed proteomic analysis. Proteomics, 2004, 4: 609-618

181 Usui K, Tomizaki KY, Mihara H. Screening of $\alpha$-helical peptide ligands controlling a calcineurin-phosphatase activity. BioOrg Medicinal Chem Lett, 2007, 17: 167-171

182 Tessier PM, Lindquist S. Prion recognition elements govern nucleation, strain specificity and species barriers. Nature, 2007, 447: 556-561

183 Syahir A, Usui K, Tomizaki KY, et al. Label and label-free detection techniques for protein microarrays. Microarrays, 2015, 4: 228-244

184 Zhao Y, Zhao X, Hu J, et al. Encoded porous beads for label-free multiplex detection of tumor markers. Adv Mater, 2009, 21: 569572

185 Xu Y, Zhang X, Luan C, et al. Hybrid hydrogel photonic barcodes for multiplex detection of tumor markers. Biosens Bioelectron, 2017, 87: 264-270

186 Appleyard DC, Chapin SC, Srinivas RL, et al. Bar-coded hydrogel microparticles for protein detection: synthesis, assay and scanning. Nat Protoc, 2011, 6: 1761-1774

187 Luan C, Xu Y, Fu F, et al. Responsive photonic barcodes for sensitive multiplex bioassay. Nanoscale, 2017, 9: 14111-14117

188 Gong X, Yan H, Yang J, et al. High-performance fluorescenceencoded magnetic microbeads as microfluidic protein chip supports for AFP detection. Anal Chim Acta, 2016, 939: 84-92

189 Liu B, Ni H, Zhang D, et al. Ultrasensitive detection of protein with wide linear dynamic range based on core-shell SERS nanotags and photonic crystal beads. ACS Sens, 2017, 2: 1035-1043

190 Xu L, Yan W, Ma W, et al. SERS encoded silver pyramids for attomolar detection of multiplexed disease biomarkers. Adv Mater, 2015, 27: 1706-1711

191 Zhao YJ, Zhao XW, Hu J, et al. Multiplex label-free detection of biomolecules with an imprinted suspension array. Angew Chem Int Ed, 2009, 48: 7350-7352

192 Hou J, Zhang H, Yang Q, et al. Hydrophilic-hydrophobic patterned molecularly imprinted photonic crystal sensors for highsensitive colorimetric detection of tetracycline. Small, 2015, 11: $2738-2742$

193 Wang H, Xu Q, Shang L, et al. Boronate affinity molecularly imprinted inverse opal particles for multiple label-free bioassays. Chem Commun, 2016, 52: 3296-3299

194 Wu S, Liu L, Li G, et al. Multiplexed detection of lung cancer biomarkers based on quantum dots and microbeads. Talanta, 2016, 156-157: 48-54

195 Bilan R, Ametzazurra A, Brazhnik K, et al. Quantum-dot-based suspension microarray for multiplex detection of lung cancer markers: preclinical validation and comparison with the Luminex xMAP' system. Sci Rep, 2017, 7: 44668

196 Liu N, Liang W, Ma X, et al. Simultaneous and combined detection of multiple tumor biomarkers for prostate cancer in human serum by suspension array technology. Biosens Bioelectron, 
2013, 47: 92-98

$197 \mathrm{Lu} \mathrm{W}, \mathrm{Fu} \mathrm{C}$, Chen Y, et al. Multiplex detection of B-type natriuretic peptide, cardiac troponin I and C-reactive protein with photonic suspension array. PLoS ONE, 2012, 7: e41448

198 Tang L, Casas J. Quantification of cardiac biomarkers using labelfree and multiplexed gold nanorod bioprobes for myocardial infarction diagnosis. Biosens Bioelectron, 2014, 61: 70-75

$199 \mathrm{Xu} \mathrm{H}$, Zhang J, Xu Y, et al. Down's syndrome screening with hydrogel photonic barcodes. Sensor Actuat B-Chem, 2018, 255: 2690-2696

200 Haasnoot W, du Pré JG. Luminex-based triplex immunoassay for the simultaneous detection of soy, pea, and soluble wheat proteins in milk powder. J Agric Food Chem, 2007, 55: 3771-3777

201 Tan A, Lim C, Zou S, et al. Electrochemical nucleic acid biosensors: from fabrication to application. Anal Methods, 2016, 8: 5169-5189

202 Deshpande A, White PS. Multiplexed nucleic acid-based assays for molecular diagnostics of human disease. Expert Rev Mol Diagnostics, 2012, 12: 645-659

203 Markou A, Strati A, Malamos N, et al. Molecular characterization of circulating tumor cells in breast cancer by a liquid bead array hybridization assay. Clinical Chem, 2011, 57: 421-430

204 Shin SH, Shin GW, Yim SH, et al. Strategy for high-fidelity multiplex DNA copy number assay system using capillary electrophoresis devices. Electrophoresis, 2011, 32: 1837-1843

205 Sankuntaw N, Sukprasert S, Engchanil C, et al. Single tube multiplex real-time PCR for the rapid detection of herpesvirus infections of the central nervous system. Mol Cellular Probes, 2011, 25: $114-120$

206 Sanghavi SK, Bullotta A, Husain S, et al. Clinical evaluation of multiplex real-time PCR panels for rapid detection of respiratory viral infections. J Med Virol, 2012, 84: 162-169

207 Deshpande A, Wheeler CM, Hunt WC, et al. Variation in HLA class I antigen-processing genes and susceptibility to human papillomavirus type 16-associated cervical cancer. J Infect Dis, 2008, 197: 371-381

208 Lau LT, Feng XY, Lam TY, et al. Development of multiplex nucleic acid sequence-based amplification for detection of human respiratory tract viruses. J Virological Methods, 2010, 168: 251254

209 Deshpande A, Gans J, Graves SW, et al. A rapid multiplex assay for nucleic acid-based diagnostics. J MicroBiol Methods, 2010, 80: 155-163

210 Hegde MR, Chin ELH, Mulle JG, et al. Microarray-based mutation detection in the dystrophin gene. Hum Mutat, 2008, 29: 1091-1099

211 Zhou WJ, Chen Y, Corn RM. Ultrasensitive microarray detection of short RNA sequences with enzymatically modified nanoparticles and surface plasmon resonance imaging measurements. Anal Chem, 2011, 83: 3897-3902

212 Kao LTH, Shankar L, Kang TG, et al. Multiplexed detection and differentiation of the DNA strains for influenza A (H1N1 2009) using a silicon-based microfluidic system. Biosens Bioelectron, 2011, 26: 2006-2011

213 Zhang Y, Zhang L, Sun J, et al. Point-of-care multiplexed assays of nucleic acids using microcapillary-based loop-mediated isothermal amplification. Anal Chem, 2014, 86: 7057-7062

214 Sha MY, Walton ID, Norton SM, et al. Multiplexed SNP genotyping using nanobarcode particle technology. Anal Bioanal Chem, 2006, 384: 658-666
215 Gao Y, Stanford WL, Chan WCW. Quantum-dot-encoded microbeads for multiplexed genetic detection of non-amplified DNA samples. Small, 2011, 7: 137-146

216 Chapin SC, Doyle PS. Ultrasensitive multiplexed microRNA quantification on encoded gel microparticles using rolling circle amplification. Anal Chem, 2011, 83: 7179-7185

217 Xu Y, Wang H, Luan C, et al. Porous hydrogel encapsulated photonic barcodes for multiplex microRNA quantification. Adv Funct Mater, 2018, 28: 1704458

218 Zhao B, Shen J, Chen S, et al. Gold nanostructures encoded by non-fluorescent small molecules in polyA-mediated nanogaps as universal SERS nanotags for recognizing various bioactive molecules. Chem Sci, 2014, 5: 4460-4466

219 Wang X, Choi N, Cheng Z, et al. Simultaneous detection of dual nucleic acids using a SERS-based lateral flow assay biosensor. Anal Chem, 2017, 89: 1163-1169

220 Zhao Y, Zhao X, Tang B, et al. Quantum-dot-tagged bioresponsive hydrogel suspension array for multiplex label-free DNA detection. Adv Funct Mater, 2010, 20: 976-982

221 He B, Morrow TJ, Keating CD. Nanowire sensors for multiplexed detection of biomolecules. Curr Opin Chem Biol, 2008, 12: 522528

222 Liu X, Wang F, Aizen R, et al. Graphene oxide/nucleic-acid-stabilized silver nanoclusters: functional hybrid materials for optical aptamer sensing and multiplexed analysis of pathogenic DNAs. J Am Chem Soc, 2013, 135: 11832-11839

223 Ma L, Hong Y, Ma Z, et al. Multiplexed highly sensitive detections of cancer biomarkers in thermal space using encapsulated phase change nanoparticles. Appl Phys Lett, 2009, 95: 043701

224 Boyerinas B, Park SM, Hau A, et al. The role of let-7 in cell differentiation and cancer. Endocrine Related Cancer, 2010, 17: F19-F36

225 Jiang L, Shen Y, Zheng K, et al. Rapid and multiplex microRNA detection on graphically encoded silica suspension array. Biosens Bioelectron, 2014, 61: 222-226

226 Hauck TS, Giri S, Gao Y, et al. Nanotechnology diagnostics for infectious diseases prevalent in developing countries. Adv Drug Deliver Rev, 2010, 62: 438-448

227 Kim J, Biondi MJ, Feld JJ, et al. Clinical validation of quantum dot barcode diagnostic technology. ACS Nano, 2016, 10: 4742-4753

228 Giri S, Sykes EA, Jennings TL, et al. Rapid screening of genetic biomarkers of infectious agents using quantum dot barcodes. ACS Nano, 2011, 5: 1580-1587

229 Ming K, Kim J, Biondi MJ, et al. Integrated quantum dot barcode smartphone optical device for wireless multiplexed diagnosis of infected patients. ACS Nano, 2015, 9: 3060-3074

230 Wang HY, Hua XW, Jia HR, et al. Universal cell surface imaging for mammalian, fungal, and bacterial cells. ACS Biomater Sci Eng, 2016, 2: 987-997

231 Wang HY, Jia HR, Lu X, et al. Imaging plasma membranes without cellular internalization: multisite membrane anchoring reagents based on glycol chitosan derivatives. J Mater Chem B, 2015, 3: 6165-6173

232 Zhang X, Chen X, Yang J, et al. Quaternized silicon nanoparticles with polarity-sensitive fluorescence for selectively imaging and killing gram-positive bacteria. Adv Funct Mater, 2016, 26: 59585970

233 Chen PJ, Hu SH, Hung WT, et al. Geometrical confinement of quantum dots in porous nanobeads with ultraefficient fluorescence for cell-specific targeting and bioimaging. J Mater Chem, 
2012, 22: 9568-9575

234 Kuo CT, Peng HS, Rong Y, et al. Optically encoded semiconducting polymer dots with single-wavelength excitation for barcoding and tracking of single cells. Anal Chem, 2017, 89: 6232-6238

235 Zavaleta CL, Smith BR, Walton I, et al. Multiplexed imaging of surface enhanced Raman scattering nanotags in living mice using noninvasive Raman spectroscopy. Proc Natl Acad Sci USA, 2009, 106: $13511-13516$

236 Lee S, Chon H, Lee J, et al. Rapid and sensitive phenotypic marker detection on breast cancer cells using surface-enhanced Raman scattering (SERS) imaging. Biosens Bioelectron, 2014, 51: 238243

237 Jimenez de Aberasturi D, Serrano-Montes AB, Langer J, et al. Surface enhanced Raman scattering encoded gold nanostars for multiplexed cell discrimination. Chem Mater, 2016, 28: 67796790

238 Macosko EZ, Basu A, Satija R, et al. Highly parallel genome-wide expression profiling of individual cells using nanoliter droplets. Cell, 2015, 161: 1202-1214

239 Klein AM, Mazutis L, Akartuna I, et al. Droplet barcoding for single-cell transcriptomics applied to embryonic stem cells. Cell, 2015, 161: 1187-1201

240 Kang WJ, Chae JR, Cho YL, et al. Multiplex imaging of single tumor cells using quantum-dot-conjugated aptamers. Small, 2009, 5: 2519-2522

241 Nwankire CE, Venkatanarayanan A, Glennon T, et al. Label-free impedance detection of cancer cells from whole blood on an integrated centrifugal microfluidic platform. Biosens Bioelectron, 2015, 68: 382-389

242 Lu J, Zheng F, Cheng Y, et al. Hybrid inverse opals for regulating cell adhesion and orientation. Nanoscale, 2014, 6: 10650-10656

243 Zhang B, Cai Y, Shang L, et al. A photonic crystal hydrogel suspension array for the capture of blood cells from whole blood. Nanoscale, 2016, 8: 3841-3847

244 Maiti KK, Samanta A, Vendrell M, et al. Multiplex cancer cell detection by SERS nanotags with cyanine and triphenylmethine Raman reporters. Chem Commun, 2011, 47: 3514-3516
245 Yang G, Liu H, Liu X, et al. Underwater-transparent nanodendritic coatings for directly monitoring cancer cells. Adv Healthcare Mater, 2014, 3: 332-337

246 Hao HC, Yao DJ. Detection of cancer cells on a chip. CTMC, 2015, 15: 1543-1550

247 Meng J, Zhang P, Zhang F, et al. A self-cleaning $\mathrm{TiO}_{2}$ nanosisallike coating toward disposing nanobiochips of cancer detection. ACS Nano, 2015, 9: 9284-9291

248 Alix-Panabieres C, Pantel K. Circulating tumor cells: liquid biopsy of cancer. Clinical Chem, 2013, 59: 110-118

249 Wang S, Liu K, Liu J, et al. Highly efficient capture of circulating tumor cells by using nanostructured silicon substrates with integrated chaotic micromixers. Angew Chem Int Ed, 2011, 50: 3084-3088

250 Zheng F, Cheng Y, Wang J, et al. Aptamer-functionalized barcode particles for the capture and detection of multiple types of circulating tumor cells. Adv Mater, 2014, 26: 7333-7338

251 Liu W, Shang L, Zheng F, et al. Photonic crystal encoded microcarriers for biomaterial evaluation. Small, 2014, 10: 88-93

252 Fu F, Shang L, Zheng F, et al. Cells cultured on core-shell photonic crystal barcodes for drug screening. ACS Appl Mater Interfaces, 2016, 8: 13840-13848

253 Andreiuk B, Reisch A, Lindecker M, et al. Fluorescent polymer nanoparticles for cell barcoding in vitro and in vivo. Small, 2017, 13: 1701582

Acknowledgements This work was supported by the National Natural Science Foundation of China (21473029 and 51522302), the NSAF Foundation of China (U1530260), the Key Medical Projects of Jiangsu Province ( BL2014078), Key Discipline of Jiangsu Province (2016-2020), the Natural Science Foundation of Jiangsu (BK20140028), and the Scientific Research Foundation of Southeast University.

Author contributions $\mathrm{Xu} \mathrm{Y}$ wrote the manuscript and all authors contributed to the general discussion.

Conflict of interest The authors declare that they have no conflict of interest. 


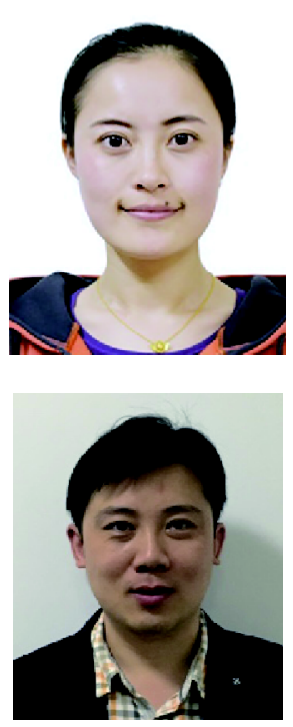

Yueshuang $\mathrm{Xu}$ is currently a $\mathrm{PhD}$ student at Southeast University and is studying as a visiting student at Stanford University in Prof. Jianghong Rao's group. Under the guidance of Prof. Yuanjin Zhao, her research focuses on the study of multiplex cancer detection based on photonic crystal barcode particles.

Yuanjin Zhao received his $\mathrm{PhD}$ in 2011 from Southeast University. He then worked as a lecturer at the State Key Laboratory of Bio-electronics. In 2012, he was promoted to be an associate professor of Southeast University. In 20092010, he worked as a research scholar at Prof. David A. Weitz's group in SEAS of Harvard University. His current scientific interests include microfluidic-based materials fabrication, biosensors, and bio-inspired photonic nanomaterials.

\section{基于新兴编码微载体的多元生物检测}

徐月霜 ${ }^{1}$, 王欢 ${ }^{2}$, 陈宝安 ${ }^{1^{*}}$, 刘宏 ${ }^{2^{*}}$, 赵远锦 ${ }^{12^{*}}$

摘要 随着对大量生物分子的高通量分析的需求不断增长, 多元分析成为用于进行大规模生物分析的前景技术. 在目前的技术中, 基于编 码微载体的液相芯片已被广泛用于如临床、医学、营养和环境等诸多研究领域的多元生物检测中. 这些编码微载体除了具有独特的编码 形式, 还具有更高的灵活性, 更好的灵敏度及更快的反应动力学. 在本综述中, 我们根据编码方法描述了一些常见的编码微载体, 并且介绍 了它们在不同靶标(如蛋白质, 核酸及细胞)的多元生物分析中的应用, 以及在不同领域(如监测食品安全, 药物研究和临床诊断)中的应用. 